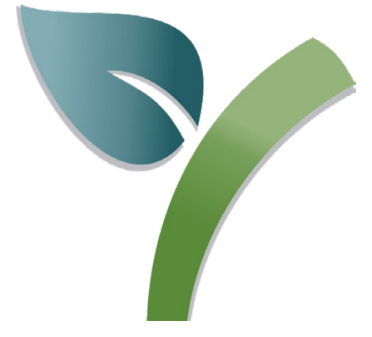

\title{
EXPLORING THE OVERLOOKED DIVERSITY OF PLANT-ASSOCIATED CERRADO MICROFUNGI
}

\author{
José Carmine Dianese ${ }^{1}$, Carlos Antônio Inácio², Anibal Alves de \\ Carvalho-Junior ${ }^{3}$, Maria do Desterro Mendes dos Santos ${ }^{4}$, \\ Taimy Cantillo-Pérez ${ }^{5}$, Danilo Batista Pinho ${ }^{6}$
}

\begin{abstract}
In this review, we present an historic account of the work on the diversity of fungi associated with plants of the Cerrado Biome, including an overview of the studies carried out by the research group of the Mycological Collection connected to the Herbarium UB (Universidade de Brasília), and the rust fungi in Herbarium IBI (Instituto Biológico, São Paulo). The contributions to Cerrado mycology from the early 19th century to the present days were highlighted. Illustrative maps were included to show the geographical distribution of the main fungal groups, associated with native plants of the Cerrado, belonging to the five predominant orders: Pucciniales (rust fungi), Phyllachorales (tar-spot fungi), Capnodiales (cercosporoid fungi: Family Mycosphaerellaceae), as well as Meliolales and Asterinales (black mildews).

KEYWORDS: black mildews-Asterinales and Meliolales, cercosporoid fungi-Mycosphaerellales, Neotropical microfungi, rust fungi-Pucciniales, tar-spot fungi-Phyllachorales
\end{abstract}

\section{INTRODUCTION}

The aim of this publication was to present and analyze data on plant-associated microfungi from Brazilian Cerrado, gathered in the Mycological Collection of Herbarium UB (Universidade de Brasília) (MCHUB) from 1993 until 2021, and the rust fungi in Herbarium IBI (Instituto Biológico de São Paulo), collected in three reserves belonging to the State of São Paulo (Mogi Guaçu, Mogi Mirim, and Antônio Carlos).

The MCHUB inventory resulted from a series of expeditions to all core states where the Cerrado biome predominates. Most of the field work was financed by a US\$260,000.00 grant to the Universi- dade de Brasília by the Fundação Banco do Brasil, which also covered costs of the physical infrastructure of the Collection. Those expeditions starting in 1994 were responsible for the inclusion of over $85 \%$ of all fungi presently deposited in the MCHUB. Extensive exploration included the main Cerrado official reserves, mainly the National Parks in Goiás, Mato Grosso, Mato Grosso do Sul, Minas Gerais, and Distrito Federal, where detailed surveys of plant-associated microfungi were for the first time the object of work by Brazilian mycologists. The result was the ex situ preservation of almost 25,000 exsiccates containing an average of two fungal species each, representing about 50,000 different fungi on

\footnotetext{
${ }^{1}$ Departamento de Fitopatologia/Biologia Celular-Biologia Microbiana, Universidade de Brasília, 70910-900, Brasília, Distrito Federal, Brazil; ${ }^{2}$ Departamento de Entomologia e Fitopatologia, Universidade Federal Rural do Rio de Janeiro, Rodovia BR 465, Km 07, s/n Zona Rural, 23890-000 Seropédica, RJ, Brazil, email-mail:carlosainacio.2009b@gmail.com; ${ }^{3}$ Instituto de Pesquisas Jardim Botânico do Rio de Janeiro, R. Pacheco Leão 915, 22460-030 Rio de Janeiro, RJ, Brasil, email:carvanibal@gmail.com; ${ }^{4}$ Departamento de Ecologia, Universidade de Brasília, 70910-900 Brasília, Distrito Federal, Brazil, email:mariadsmendes@gmail.com; ${ }^{5}$ Universidade Estadual de Feira de Santana, Novo Horizonte, 44036900 Feira de Santana, Bahia, Brazil, email: taycantillo@gmail.com; ${ }^{6}$ Departamento de Fitopatologia, Universidade de Brasília, 70910-900, Brasília, Distrito Federal, Brazil, email: danilopinho@unb. br. Corresponding author: jcarmine@gmail.com
} 
herborized plant material preserved in envelopes measuring $30 \times 20 \mathrm{~cm}$. This large volume of material allows for the subsampling of each exsiccate to study the different fungi found in each one. The collection inventory includes among members of the kingdom Fungi, some saprotrophic Agaricomycetes (Agaricales, Boletales, Cantharellales, Corticiales, Geastrales, Hymenochaetales, Polyporales, Russulales) that make up mere 311 exsiccates, out of a total of 24,572. However, the focus of the MCHUB are the microscopic fungi associated with the leaves and branches of the native plants from Cerrado, which include epiphytes, parasites, and hyperparasites. These microfungi are distributed in phylum Ascomycota among 7 classes, 42 orders and 47 families, and for the phylum Basidiomycota in 8 classes, 11 orders, and 27 families. Among microfungi belonging to Ascomycota and Basidiomycota a total of 744 genera are represented in the MCHUB, with a total of 642 identified at the species level. The inventory is in a dynamic phase where there is material available and space for additional taxonomical effort.

This publication will reveal the result reached up to now, that include collections from 1991 until 2021, at the main body of the Cerrado biome. However, the Cerrado in the State of São Paulo and the Cerrado islands of the State of Roraima were not exploited by the MCHUB expeditions, and unfortunately regions in the States of Piaui and Tocantins presently remains subsampled. However, the important data on rust fungi (Pucciniales) from São Paulo, contributed by Carvalho-Junior et al. (2008), was included in our analyses. It is not our objective to review all the mycological studies and taxonomical novelties revealed by the work at the MCHUB, but simply list the available information, review historical aspects of the Cerrado mycology, and show the geographical distribution of the predominant microfungal groups found in the Cerrado. These main groups are mostly biotrophic, host specific plant parasites, and will be threatened with extinction whenever this happens to their plant hosts.

\section{THE CERRADO}

The Cerrado is a Neotropical savanna that occupies about 2 million $\mathrm{km}^{2}$, or $22 \%$ of the Brazilian territory, and is in the heart of South America. Among Brazilian biomes, the Cerrado is second in extension, smaller only than the Amazonian biome, and extends over the states of Bahia, Goiás, Mato
Grosso, Mato Grosso do Sul, Minas Gerais, Maranhão, Piauí, São Paulo, Tocantins, Federal District, with large islands of vegetation in Roraima (CAVALCANTI \& JOLY 2002). Additionally, it encroaches into the neighboring countries, Bolivia, and Paraguay (VELAZCO 2018), where a fungal collection effort was never performed and is badly needed. Furthermore, Velazco (2018) argued that the highest plant species richness and rarity are found in the central part of the Brazilian Cerrado, which is, at the same time, the most disturbed region. However, Bolivia is the second country showing large undisturbed areas with high species richness and rarity, mainly associated with the Cerrado and the Chiquitana Dry Forest. In Paraguay, plant species richness and rarity were concentrated mainly in the northern part of the Chaco Humido and Chaco Seco. These are two countries where a collaborative effort is needed to begin exploring fungal diversity in substantial fraction of the Cerrado biome outside of the Brazilian frontiers.

The Brazilian Cerrado has over 12,000 known plant species, of which $44 \%$ are endemic (SCARIOT et al. 2005, MENDONÇA et al. 2008, MARTINELLI \& MORAES 2013), and 645 species are critically endangered. This represents more than $30 \%$ of the species that are on the red list of threatened species in Brazil (MARTINELLI \& MORAES 2013). Almost all Brazilian herbaceous plants or herbs occur in this region (SCARIOT et al. 2005). Furthermore, Lima (2011) showed that the headwaters of the biome contribute to feed eight of the 12 Brazilian hydrographic regions as defined by the National Water Agency - ANA. Consequently, the importance of the biome to maintain the country's water resources is significant. Giulietti et al. (2009) list 2,291 species of species of rare plants in Brazil, meaning that around $7 \%$ of our flora is rare, the majority of which is concentrated in Cerrado areas. In Brazil, there are more than 43,000 indigenous vascular plants (GIULIETTI et al. 2005, 2009). Considering a global flora of $c a$ 380,000 species, about 14 to $15 \%$ of world plant diversity is present in Brazil (PATON et al., 2008). Expansion of modern agriculture, bad management of the tropical forests, and deforestation in areas where endemism occurs in high levels, led to the establishment of thirty-five global biodiversity hotspots, two of them in Brazil, Cerrado and Atlantic Forest hotspots (MITTERMEIER et al. 1998, MYERS et al. 2000, STRASSBURG et al. 2017), with endemism estimated in 4,400 species for the Cerrado, and 8,000 
for the Atlantic Forest. Many of the plant associated microfungi are mostly parasitic and thus depend on their host to survive. Thus, whenever a plant enters the list of endangered species a set of microfungi are also at risk of extinction. It was shown that over ten fungal species can be associated with each plant species in the Cerrado (DIANESE 2000). Thus, just in the case of the 4,400 endemic species, we estimated an associated mycota containing over 40,000 fungal species. As just around 1,000 such fungi were reported from the Cerrado, it is clear the need to accelerate efforts on ex situ conservation through the implementation of stable culture collections to preserve those culturable species growing on Cerrado plants. This is a basic step and a great challenge if we want to explore our mycodiversity for the benefit of the country and the world. However, before that we need to identify our microfungi taking them out of their generalized anonymity, we need to name them. This is where MCHUB contributes with Cerrado mycology by practicing a taxonomical work with credibility, as recognized internationally. Finally, the continuous use of the phylogenetic species concept for the identification of every specimen studied, maintaining the continuity of field work to obtain fresh material, and an effort to expand the culture collection, will ensure future academic growth for the MCHUB.

\section{A BRIEF HISTORY OF CERRADO MYCOLOGY}

During the ninth Century, there were practically no mycological studies carried out by Brazilians in Brazil. However, contributions to the knowledge of the Brazilian fungi started with works by European mycologists focusing macromycetes. Fidalgo (1968) highlighted important contributions starting with Link (1809) recording six species of basidiomycetes from Brazil. The Rev. Miles Joseph Berkeley published a series from 1844 to 1856 , designated as Decades of Fungi, that included descriptions of fungi from South America, namely from Peru, and Rio Negro in Brazil (PEGLER 1996).

Berkeley (1843) covered a wide group of macromycetes from Brazil provided by the Scottish medical doctor and important plant collector George Gardner, who lived in Brazil from 1836 to 1841 (GARDNER 1849). Gardner collected extensively along the coastal areas of the Brazilian Atlantic Forest, with a few specimens of fungi from the States of Goiás and Minas Gerais where Cerrado vegetation predominates (GARDNER 1840). Among ascomycetes, only a sooty mold (Antennaria pannosa) was described, and Cladosporium herbarium listed (BERKELEY 1843). Later, Berkeley \& Cooke (1877) reported that 437 species of mostly macromycetes were known from Brazil, including 356 Hymenomycetes, 55 Ascomycetes and 13 Gasteromycetes. Berkeley (1879) reported on 81 species collected by the French botanist A.F.M. Glaziou (FUNDAÇÃO CASA DE RUI BARBOSA 2021) from the Atlantic Forest at Rio de Janeiro. Cooke (1892) described a few ascomycetes on plants and plant debris from Brazil (Helotiella stromatica (=Puttemansia stromatica, Dothidella bambusae (=Rhopographus bambusae), Clypeolum dissiliens (=Schizopeltis dissiliens), and Metasphaeria pusilla).

A historical highlight was the arrival of Carlos Luiz Spegazzini (*1858-†1926) in Argentina in 1879, an Italian immigrant who became the most important Latin American mycologist, as shown by Farr (1973). He collected, described, and established the first collection of microfungi of South America, that remains as the most important today, followed by those established much later by Ahmés Pinto Viégas in the 40ies, and Augusto Chaves Batista starting in 1951. Most fungi from Brazil studied by Spegazzi$\mathrm{ni}$, were obtained by the Catalan collector Puiggari from the Atlantic Forest in Apiaí, State of São Paulo (SPEGAZZINI 1881abcd, 1889), and by Alfred Usteri (GLOBAL PLANTS 2021), a Swiss botanist who spent part of his life also in São Paulo, including for the first time areas covered by the Brazilian Cerrado. Spegazzini (1908), working with Usteri's collections, established important ascomycete genera (e.g. Dimerosporiella, Dimeriella, Eudarluca, Hyalotheles, and Phaeoseptoria), including some hyperparasites (species of Dimerosporium, Dimeriella, Eudarluca, Lonchospermella, Zukalia), six new Cercospora species, four new Phyllachora, four new Septoria species, and two new Meliola species.

However, the plant-associated Cerrado mycobiota remained practically unexplored, and had its most definitive beginning with the works by Paul Christopher Hennings (*1841-†1918), an eminent German mycologist working at the Herbarium Berolinense, firmely connected in Brazil through the German botanist naturalized Brazilian Ernst $\mathrm{H}$. G. Ule (GLOBAL PLANTS 2021). Working with Ule collections from 1883 to 1912, Hennings studied the mycobiota associated with primeval Cerrado 
vegetation present in the States of São Paulo (HENNINGS 1896), Goiás (HENNINGS 1895), Mato Grosso (HENNINGS 1900), Minas Gerais (HENNINGS 1896). Hennings (1902ab, 1904ab, 1908) was also supplied with important collections by the Belgian Brazilian plant-pathologist Arsènne Putemans (PATRIMÔNIO BELGA NO BRASIL 2021). Putemans deserves also to be remembered as the first collector to put together a fungarium in Brazil, today located at Herbarium of the Universidade Federal Rural do Rio de Janeiro UFRRJ, presently curated by Prof. Carlos A. Inacio (NYBG STEERE HERBARIUM 2021).

Paul C. Hennings gave a relevant contribution to the early knowledge of the Cerrado microfungi from 1895 to 1908, predominantly from areas in the State of Goiás, with a few samples from Minas Gerais and São Paulo, describing a total of 143 species in 42 genera of Ascomycota, 6 Pucciniales, and 1 Ustilaginales (Aposphaeria glaziovii, Asterina salaciae, Asteroma tecomae, Campsotrichum tetracerae, Coniothyrium salaciae, Dicoccum glaziovii $\equiv$ (=Septoidium glaziovii), Dimerosporium echites (三 Seynesia echites), Dothidella glaziovii, Excipula ilicicola, Helminthosporium tapurae, Ophiobolus ingae (三 Diatractium ingae), Ophiobolus ingae (= Diatractium ingae), Phloeospora myrtacearum, Phoma cocoes, Phyllachora ficicola, Phyllachora urbaniana, Phyllosticta clusiae, Phyllosticta cocoes, Phyllosticta kielmeyerae, Phyllosticta lafoensiae (三 Asteromella lafoensiae), Phyllosticta pterandrae (三 Asteromella pterandrae), Phyllosticta qualeae (三 Asteromella qualeae), Phyllosticta qualeae (E Asteromella qualeae), Placosphaeria cordiae, Placosphaeria salvertiae, Placosphaeria smilacis, Placosphaeria vochysiae, Septorella salaciae, Septoria symploci.

In addition, other German Botanist and Mycologist Andreas Allerscher (*1828-†1903) described 30 species of ascomycetes on Cerrado plants, working with collections from the State of Goiás by the French botanist August Glaziou (FUNDAÇÃO CASA DE RUI BARBOSA 2021), a report included in Hennings (1895).

Dietel $(1897,1899)$ studying Ule's collections of rust fungi described 70 species from Brazil, however, only six from the Cerrado of Minas Gerais: Aecidium guatteriae, Puccinia circinans (=P. arenariae), $P$. velata, Uredo alstroemeriae. U. uleana, Uromyces giganteus ( $=U$. brasiliensis).

Anders Fredrik Regnell (*1807-†1884) a Swedish medical doctor that just after graduation in 1837 in Stockholm, moved to Brazil and settled in the small town of Caldas in the State of Minas Gerais, until his death in 1884 . There, besides his excellent medical work, he became a self-taught botanist, and one of the most important plant collectors of the country. He was a highly regarded medical doctor, and had also significant investments in coffee plantations, yielding him a large fortune also as a moneylender, practicing interests of up to $20 \%$ a year (SANTOS 2016). He financially supported a group of Swedish botanists and collectors in Brazil, and together they gave an enormous contribution to the study of the Brazilian flora, that included some fungi (CONCHA-QUEZADA 2012). The Royal Swedish Academy of Sciences, in 1870, 14 years before Regnell's death established the Regnellian Herbarium at the Swedish Museum of Natural History to accommodate the Brazilian collection, today with 11,500 plant specimens, containing 7,500 nomenclatural types from Brazil. The Regnellian Fund created with resources originating from the fortune donated by Regnell remains still active (SANTOS 2016).

A few species of rust fungi from the Cerrado in the State of Mato Grosso, collected by RegnelI's partners, C.A.M. Lidman and G.O. Malme, were described by the Swedish mycologist Juel (1897), as follows: Aecidium calosporum and Uromyces foveolatus, collected by Malme; and Aecidium mattogrossense, A. momordicae, Leptinia brasiliensis (type species of Leptinia), Uredo mogiphanis, based on Lidman's collections. Later Jørstad (1956) contributed with the descriptions of Crossopsora angusta, Puccinia callosa, both holotypes collected by Malme.

Major contributions specifically to the study of Cerrado rust fungi came from the American explorer and banker E. D. W. Holway, who preferentially collected rust fungi extensively in South America during the early 1920's (ARTHUR 1925). His collections were the raw material for important works published later by H. S. Jackson that included the description of 16 species of Pucciniales from the Cerrado (JACKSON 1926, 1927, 1931abc, 1932).

Thurston-Junior (1940) studied several rust fungi mostly from the Atlantic Forest, but included two new species, Endophylloides duguetiae, recognized as Dietelia duguetiae by Buriticá \& Hennen (1980), and Uredo cassiae-rugosae, both from the Cerrado at Uberlândia, Minas Gerais.

In 1912, the French mycologist André Pierre 
Jules Maublanc (*1880-†1958) worked with Eugenio dos Santos Rangel (*1877-†1953) at Museu Nacional in Rio de Janeiro (MAUBLANC 1913, MAUBLANC \& RANGEL 1915, GRILLO 1935). Rangel (1916a, 1916b, 1918, 1921) became the first native Brazilian to work with taxonomy of microfungi, concentrated mainly in the study of pathogenic fungi on crop plants, and those associated with plants common in both Atlantic Forest and Cerrado, as the case of fungi in Tabebuia (=Handroanthus) species (RANGEL 1921, DIANESE et al. 1994b).

The two most important Brazilian mycologists, Batista (*1916-†1967) (CARNEIRO 1968, BEZERRA et al. 2017) and Viégas (*1905-†1986) (COSTA 1986) benefitted from the fungal collections of Cerrado microfungi by the pioneer botanist and first Emeritus Professor of the Institute of Biological Sciences of the University of Brasilia, the late Prof. Ezechias Paulo Heringer (*1905-†1987). E. P. Heringer, the founding botanist and ecologist of Brasília, the new Brazilian capital inaugurated in 1960, installed over 1,000 miles inland deep into the Cerrado of Central Brazil. He was responsible for the preservation of almost 60,000 ha of Cerrado when he established the Parque Nacional de Brasília (Brasília National Park), Estação Ecológica de Águas Emendadas, an important ecological reserve, and the area where is today the Jardim Botânico de Brasília (Brasília Botanical Garden). He was honored with a natural reserve (PARQUE ECOLÓGICO EZECHIAS HERINGER 2021) and an Herbarium (JARDIM BOTÂNICO DE BRASÍLIA 2021), both named after him. He was one of the founders of the Sociedade Botânica do Brasil (2021) and its Vice-President in 1978.

After the works by the European and American collectors and mycologists, only by the early 1940's Viégas (1943, 1944, 1945bcdef, 1946) and Viégas \& Teixeira (1945) established a monumental contribution to Brazilian mycology. Viégas studied the cercosporoid fungi (VIÉGAS 1945a), later incorporated in Chupp (1953), and contributed with the description of many other Ascomycetes (VIÉGAS 1944, 1945b, 1946abc; VIÉGAS \& CARDOSO 1944) from Brazil. Viégas and collaborators revealed species characteristically associated with hosts endemic or common in the Cerrado, e.g.: Septoidium didymopanacis, Septoria cremasti, S. lafoensiae, S. siparunae, Colletotrichum cecropiae, Pestalotia rapanea, Cladobotryum australe, Passalora eragrostidis, Rhinotrichum alterosum, Spondylocladium nigerri- mum, Melanconium nectandrae, Patouillardiella copaiferae (VIÉGAS 1945a, VIÉGAS \& TEIXEIRA 1945, VIÉGAS 1947). Viégas (1961) published the historical Índice dos Fungos da América do Sul, extremely precise and useful in times that predated internet, digital records and the personal computers. Later he wrote an 890-page dictionary of mycology and plant pathology (VIÉGAS 1979). Importantly, seven years before his retirement, he described the genus Porotenus, a member of the Pucciniales from the Cerrado (VIÉGAS 1960), the only genus by a Brazilian mycologist appearing in the second edition of the Illustrated Genera of Rust Fungi (CUMMINS \& HIRATSUKA 1983).

Historically, Batista and collaborators gave the most extensive contribution to Brazilian mycology (SILVA \& MINTER 1995). Their focus in Cerrado microfungi was entirely dependent on Heringer's fieldwork starting in the mid 1950's in Paraopeba, Minas Gerais, and after 1960 in the Federal District, during the construction of Brasília. Heringer, besides his botanical and ecological work was an efficient fungal collector in those two distinct areas covered with Cerrado vegetation. In 1951, Batista inaugurated the historical Instituto de Micologia do Recife, where he became the main protagonist of ca 700 papers dealing with over 3,000 fungi in just 16 years (SILVA \& MINTER 1995, BEZERRA et al. 2017). The complete collection of Batista's full papers that include many species from the Cerrado, is freely retrievable from http://batista.fungibrasil. net/articles. With the transfer of Heringer to Brasilia in 1960, the cooperation was intensified including new collections of plant-associated microfungi (ascomycetes and rust fungi), until the passing of 51 years old Batista in 1967 (CARNEIRO 1968). Still, the result was major scientific contribution that remains as an indispensable foundation for the development of mycology in the Cerrado (BATISTA et al. 1962, 1966abc, 1967; BATISTA \& PERES 1964, 1965, 1966), with a record of 240 taxa recognized on Cerrado plants, as listed in Dianese et al. (1997). A large set of new ascomycetes were described by Batista and collaborators on material collected by Heringer, from the Cerrado in Paraopeba (State of Minas Gerais), and Distrito Federal, as follows: Ainsworthia psidii, Akaropeltis macheriifolii, Akaropeltopsis machaeriifolii, Asteromella compositarum, A. heringeri, A. ourateae, Bactrodesmiella aspidospermatis [= Pseudocercospora aspidospermatis (DIANESE \& CÂ- 
MARA 1994)], B. tecomae, Barnettia byrsonimae, $B$. lucumae, Calothyriolum brasiliae, C. brasiliense, $\mathrm{Ca}$ marosporium anacardii, Camptomeris cassiae, Cercospora roupalae, C. annonifolii, C. cnidoscolifolii, $C$. mataybae, C. vochysiae, Chaetodiplodina cubensis, Chaetophoma clitoriae, Ciferriusia piperis, Coniothyrium hypericin var. macrospora, C. mikaniae, Coremium rhopalinum, Dicoccum anacardii, Didymella smilacina, Eudimeriolum aspidospermatis, Hendersonia annonicola, H. salaciae, Leptosphaeria mikaniae, Macrophoma brasiliensis, Microdiplodia andirae, Mycosphaerella byrsonimae, M. conspicua. $M$. discophora var. macrospora, M. machaerii, M. melastomatacearum, M. smilacifolii, M. tocoyenae, $M y$ sia combreti, Periconia echinochloae, Pestalotiopsis acrocomiorum, P. annonae, Phaeoseptoria tecomae, $P$. roupalae, Phomachora anacardiicola, P. erythroxyli, Phyllachora pouteriae, P. qualeae, P. rhopalina var. macrospora, Phyllachora truncatispora var. macrocarpa, Phyllachora whetzelii var. macrospora, Phyllosticta acrocomiicola, P. lucumae, P. sizygii, Piricauda byrsonimae, Placosphaeria mauritiae, $P$. neeae, Plectopycnis bignoniacearum, Plenotrichella perseae, Pycnomyces diptericis, Rosenscheldiella rapaneae, Septoria sapindacearum, Sphaerodothis diplothemiifolii, Sphaeropsis myrtaceicola, Staibia connari, Stomiopeltis tetrasperma, Tegaster protiicola, Tegoa tabebuiae, Tuberculina phyllachoricola, Vonarxella diptericis (http://batista.fungibrasil.net/ articles, SILVA \& MINTER 1995).

Contemplating a remarkable biodiversity hotspot with over 12,000 plant species extending for over 2.2 million sq. km. (MENDONÇA et al. 2008), inspired by the enthusiasm of dedicated students, the Mycological Collection of Herbarium UB (MCHUB) was conceived in 1993 (DIANESE et al. 1997), whose contribution to Cerrado mycology is herein outlined.

CONTRIBUTION OF THE MYCOLOGICAL COLLECTION OF HERBARIUM UB - MCHUB TO THE STUDY OF CERRADO MICROFUNGI

The MCHUB is part of the history of the Instituto de Ciências Biológicas of the Universidade de Brasília (UnB) founded in 1962 (DIANESE 2014, DIANESE \& CAFÉ-FILHO 2014, DINIZ \& DIANESE 2014). Starting with a US\$260,000.00 grant to the UnB by the Banco do Brasil Foundation, in 1993, the Mycological Collection of Herbarium UB (MCHUB) remains dedicated exclusively to the study of micro- fungi associated with native plants from the Cerrado, the second most important Brazilian biodiversity hotspot (MITTERMEIER et al. 1998; MYERS et al. 2000; MYERS 2003). Twelve years later, in 2005, the MCHUB staff was responsible for the $V$ Latin American Mycological Congress. In 2010, as part of the commemoration of Brasilia's $50^{\text {th }}$ Year Anniversary, the MCHUB organized the VI Brazilian Congress of Mycology, with participation of many international mycologists (DIANESE \& SANTOS 2010). From 1993, for the last 27 years, the nationwide assessment of the Cerrado mycodiversity by the MCHUB covered epiphytes, parasites, and hyperparasites on leaves of native plants from the Cerrado. These unique collections included mainly Ascomycetes (asexual and sexual morphs), Pucciniales, and a few Ustilaginales in Brazilian national parks located in the Cerrado biome, in other private or official protected areas, and in private sites, such as legally enforced reserves in farms. Dianese et al. (1997), in a review on the Cerrado microfungi already listed a total of 834 species then known to occur in the Cerrado biome that included major contributions by Augusto Chaves Batista (SILVA \& MINTER 1995), and Viégas (1943,1944, 1945ac, 1946a).

The MCHUB field surveys for the first time covered the entire Cerrado biome in Central, Southeastern and Northeastern Brazil in a countrywide scale (Figure 1), except for three reserves located in the State of São Paulo, and the Cerrado fragment in the State of Roraima at the extreme North of the country. Hennen and collaborators (CARVALHO-JUNIOR et al. 2008) surveyed in detail the rust fungi in the state of São Paulo, and their data on Cerrado is here incorporated. The MCHUB inventory presently counts 24,572 herborized specimens that also includes ca 1,000 myxomycetes. The collection is well preserved, but needs additional taxonomic work, presently with about $20 \%$ of the specimens identified to species level.

The MCHUB activity generated in the last 26 years 11 D.Sc. thesis, 17 M.Sc. dissertations, and 65 papers published in prestigious journals dedicated to mycology and plant pathology (Mycologia, Mycological Research, Mycological Progress, IMA Fungus, Phytotaxa, Sydowia, Plant Disease, Plant Pathology), with cover illustrations in Mycologia (GUTERRES et al. 2018) and Plant Disease (AGRA et al. 2018).

Starting in 1993 the material incorporated into the MCHUB was steadily being worked through, 


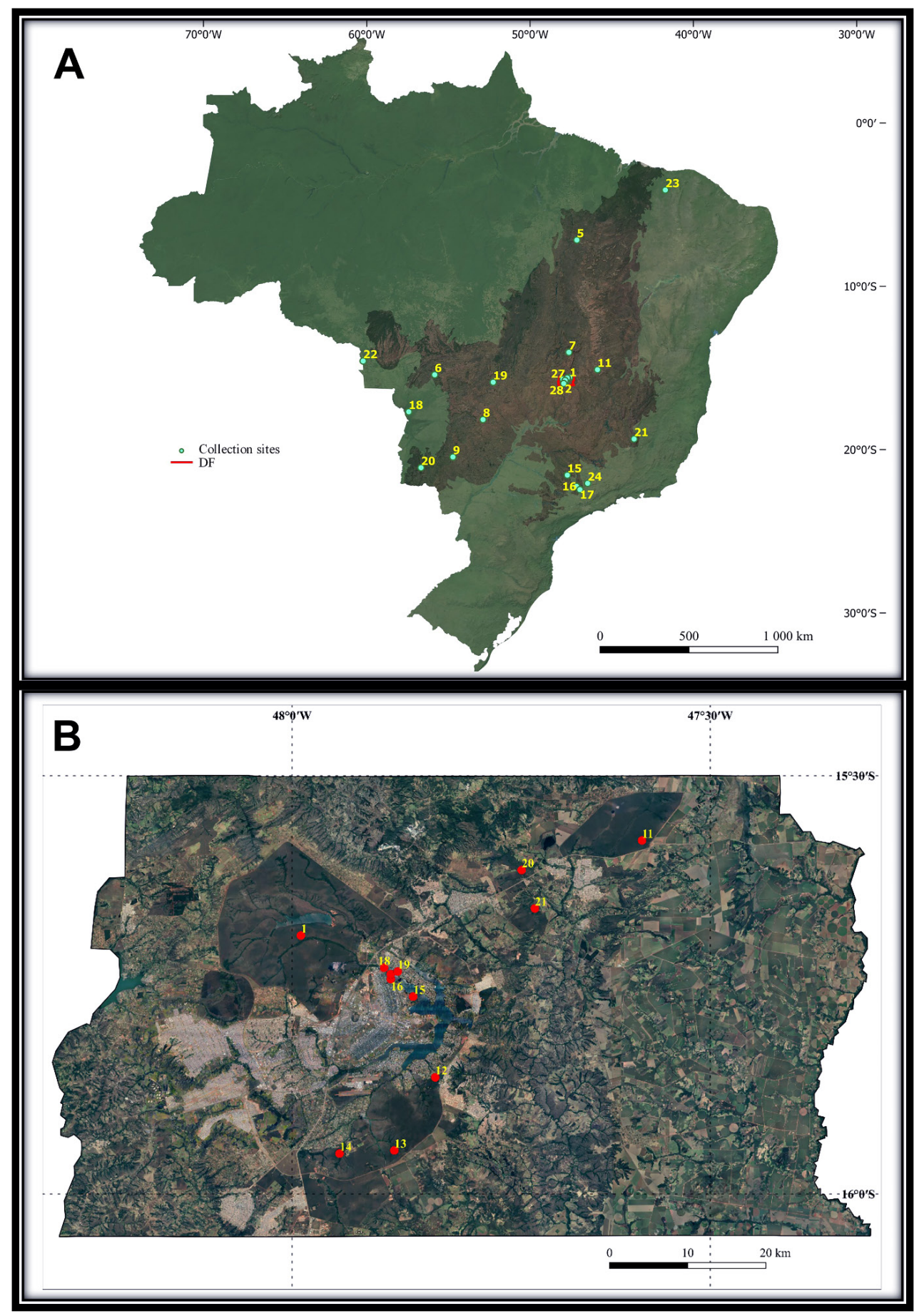

Figure 1. Twenty-eight collection sites within the Brazilian Cerrado in Brazil (A) and Federal District (B) where fungal specimens were recovered from 1993 to 2021, to construct the 24,572-voucher inventory of the MCHUB, plus the rust fungi collected in three Cerrado reserves at the State of São Paulo (CARVALHO-JUNIOR et al. 2008). Collection sites numbered from 1 to 28 in alphabetical order, with indication of the state where the collection site is located are: 1. Águas Emendadas Ecological Station-DF, 2. Brasília Botanical Garden-DF, 3. Brasília Federal Institute Cerrado Reserve, Planaltina-DF, 4. Brasília National Park-DF, 5. Chapada das Mesas National Park-MA, 6. Chapada dos Guimarães National Park-MT, 7. Chapada dos Veadeiros National Park-GO, 8. Emas National Park-GO, 9. Embrapa Beef and Catle Cerrado Reserve at Campo Grande-MS, 10. Embrapa Cerrados Natural Reserve-DF, 11. Grande Sertão Veredas National Park, Arinos-MG, 12. IBGE Ecological Reserve-DF, 13. Lago Norte Residencial Area, Brasília-DF, 14. Lago Norte Park II, Brasília-DF, 15. Luis Antônio Experimental Station-SP, 16. Mogi-Guaçu Experimental Station-SP, 17. Mogi-Mirim Experimental Station-SP, 18. Pantanal Matogrossence National Park, 19. Serra Azul State Park, Barra do Garça-MT, 20. Serra da Bodoquena National Park-MT, 21. Serra do Cipó National Park-MG, 22. Serra Ricardo Franco State Park-MT, 23. Sete Cidades National Park-PI, 24. UFU* Panga Ecological Station, 25. UnB** Arboretum, 26. UnB Biological Experiment Station, 27. UnB Campus Darcy Ribeiro, 28. UnB. *Universidade Federal de Uberlândia **Universidade de Brasília 
and when over 13,000 specimens were identified as shown in Table 1, five orders predominate, and their geographical distribution will be illustrated in a sequence of maps covering Pucciniales, Capnodiales/ Mycosphaerialles, Asterinales, Meliolales and Phyllachorales (Figures 3 to 7). These are all fungi with parasitic ability and in general with high host specificity, showing easily identifiable signs and symptoms in the field. However, as they predominate in an orderly way in such a large sample it is expected that the data would reflect their relative importance in the Cerrado when it comes to leaf associated fungi.

Historically, the plant-associated mycological research at the Universidade de Brasilia predates the MCHUB with a significant event. This highlight involves the first international publication focusing Austropuccinia psidii (=Puccinia psidii) on Eucalyptus species (DIANESE et al. 1983, 1984), a fungus that is found in the Cerrado infecting native Myrtaceae as Campomanesia adamantium, Eugenia dysenterica, E. florida, Marlierea sp., Myrcia linearifolia, Plinia cauliflora, and Psidium guajava (SOUZA 2016). In C. adamantium, plants were simultaneously infected by $A$. psidii and by Phakpsora rossmaniae, (DIANESE et al. 1993d). The disease caused by A. psidii on Eucalyptus, previously known in Brazil (FERREIRA
1989), was for the first time reported abroad at the International Congress of Plant pathology in Melbourne in 1983 (DIANESE et al. 1983) and published in the following year (DIANESE et al. 1984). These publications resulted in a historical alert bulletin from the Australian Quarantine illustrating the disease symptoms on Eucalyptus and other Myrtaceae from the Americas (NAVARATNAM 1985) (Figure 2). However, 23 years later the biological invasion by that rust fungus was confirmed in Hawaii (UCHIDA et al. 2006), four years before Australia (CARNEGIE et al. 2010). Austropuccinia psidii became a major economic problem upon reaching Australian plantations of Eucaliptus five years later (CARNEGIE 2015), and an ecological disaster with 45 myrtaceous species in decline starting in 2018 (PEGG et al. 2018). The fungus is already damaging over 375 species of native Myrtaceae in Australia and showing extreme virulence towards Rhodamnia rubescens and Rhodomyrtus psidioides (FERNÁNDEZ-WINZER et al. 2019). Similar problems are being revealed in New Zealand (SUTHERLAND et al. 2020, TOOME-HELLER et al. 2020), after the detection of the fungus in 2017 (GUY \& BARRY 2017), as predicted by Teulon et al. (2015).

The intercontinental spread of $A$. psidii, given

Table 1. Distribution among orders of the exsiccates deposited in the MCHUB that have already been studied.

\begin{tabular}{|c|c|c|}
\hline Order/Family & $\begin{array}{c}\text { Number of specimens } \\
\text { studied }\end{array}$ & $\begin{array}{c}\text { \% Specimens } \\
\text { studied }\end{array}$ \\
\hline Pucciniales & 2,603 & 19.7 \\
\hline Phylachoralles & 2,501 & 18.9 \\
\hline Capnodiales-Mycosphaerellaceae & 1,864 & 14.1 \\
\hline Meliolales & 836 & 6.3 \\
\hline Asterinales & 569 & 4.0 \\
\hline Pleosporales & 487 & 3.6 \\
\hline Erysiphales & 475 & 3.6 \\
\hline Microthyriales & 325 & 2.5 \\
\hline Diaporthales & 324 & 2.4 \\
\hline Parmulariales & 207 & 1.6 \\
\hline Hypocreales & 137 & 1.0 \\
\hline Botryosphaeriales & 91 & 0.7 \\
\hline Members of 45 other orders with a maximum of 50 specimens studied & 2,010 & 15.6 \\
\hline Total & 13,231 & 100 \\
\hline
\end{tabular}




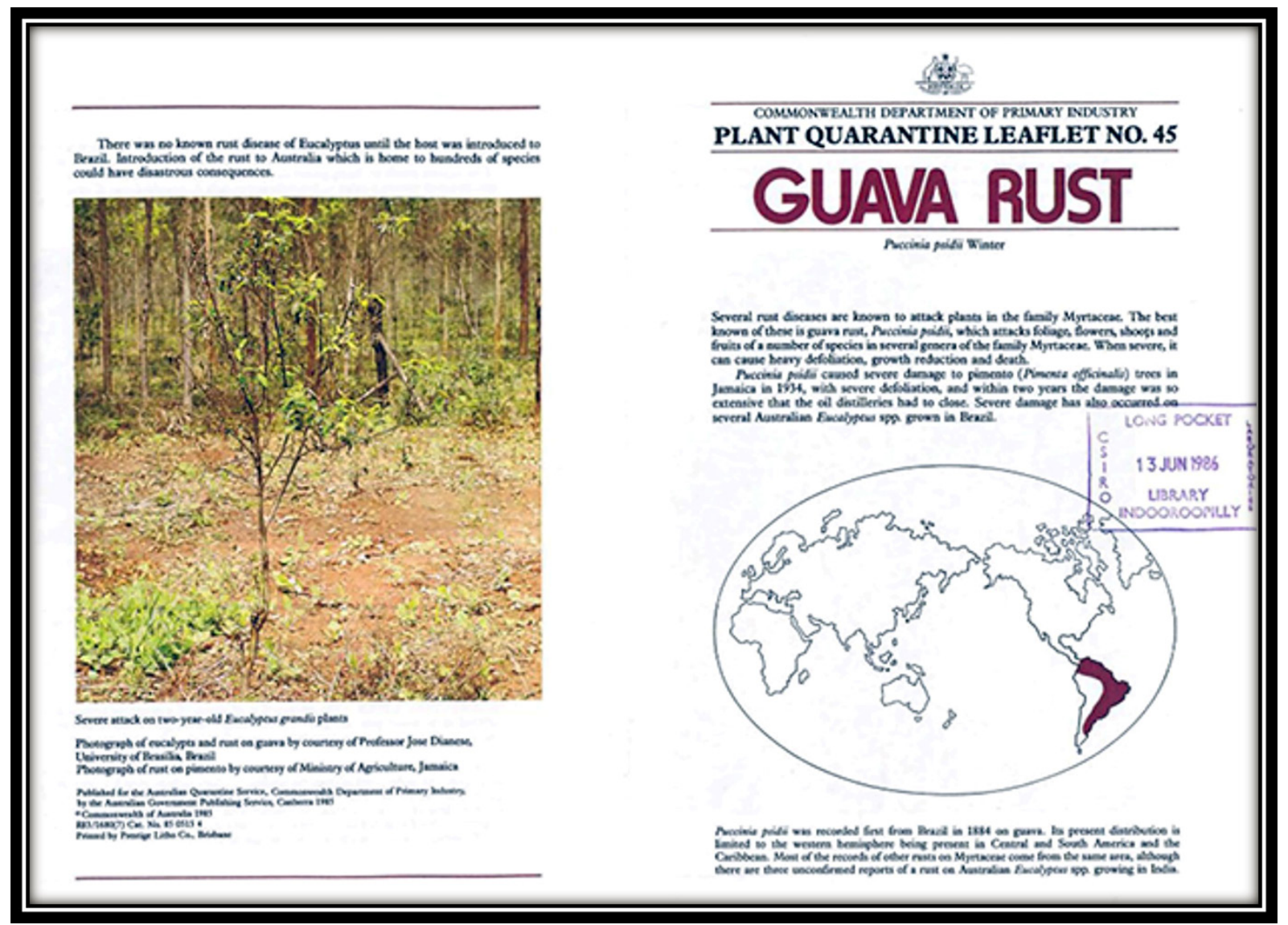

Figure 2. Historical leaflet issued by the Australian Quarantine in 1985, as a first step of a campaign to prevent introduction of Austropuccinia psidii, based on report by Dianese et al. $(1983,1984 b)$.

its broad host spectrum, is the most dramatic example of a massive biological invasion of continents by a rust fungus; from South America spreading through Central and North America, Hawaii, Southeast Asia, Australia, and New Zealand. The first work on the epidemiology of $A$. psidii (TESSMANN et al. 2001) published internationally was released 8 years after the inauguration of the MCHUB.

\section{STUDIES ON CERRADO RUST FUNGI}

In the second edition of the classic manual, Illustrated Genera of Rust Fungi, by Cummins \& Hiratsuka (1983), the only genus by a Brazilian mycologist was Porotenus described 23 years before (VIÉGAS 1960). The contributions of the MCHUB expanded this participation in the new 2003-edition of the Illustrated Genera of Rust Fungi. Cummins \& Hiratsuka (2003) accepted 105 genera of Pucciniales, with the significant addition of two new genera from the Cerrado, Batistopsora, type species $B$. crucis-filii (DIANESE et al. 1993d), and Kimuromyces, type species $K$. cerradensis (DIANESE et al. 1994d), plus the reinstatement of the genus Mimema based on Mimema venturae (DIANESE et al. 1995). Taxono- mic novelties of Cerrado rust fungi with holotypes deposited in the MCHUB were included in Table 2.

The entire inventory of the Cerrado rust fungi already identified and deposited in the MCHUB was shown in the Table 3.

Presently, 112 species of rust fungi are known to possess holotypes collected in the Cerrado as registered in the MCHUB and listed by Hennen et al. (2005) and Souza (2016). These fungi belong to 27 different genera being 18 Puccinia spp., 17 Ravenelia spp., 15 Uromyces spp., 12 Aecidium spp., 9 Prospodium spp., 7 Uredo spp., 6 Phakopsora spp., $4 \mathrm{Ce}$ rotelium spp., 4 Crossopsora spp., 2 Chaconia spp., 2 Porotenus spp. The remaining 16 genera (Aplopsora, Batistopsora, Catenulopsora, Cerradoa, Cionothrix, Crossopsorella, Dicheirinia, Didymopsora, Dietelia, Diorchidiella, Diorchidium, Kimuromyces, Leptinia, Mavavalia, Mimema, Neopuccinia, and Skierka) have one species originally collected in the Cerrado.

The rust fungi known for the entire Cerrado biome were described from six Brazilian states: Bahia (Ravenelia corbuloides, R. tortuosa); Goiás (Aecidium annonae, A. byrsonimaae, A. invallatum, A. meiapontense, A. vinnulum, A. xylopiae, Ciono- 
Table 2. Pucciniales from the Cerrado biome described from 1993 with holotypes deposited in the Mycological Collection of Herbarium UB- MCHUB.

\begin{tabular}{|c|c|}
\hline SPECIES / YEAR & SOURCE \\
\hline Aplopsora hennenii / 1995 & 1 \\
\hline Batistopsora crucis-filli / 1993* & 2 \\
\hline Cerotelium giacometii / 1993 & 2 \\
\hline Crossopsora hymenaeae / 1994 & 3 \\
\hline Crossopsorella byrsonimae / 2018* & 10 \\
\hline Kimuromyces cerradensis / 1995* & 5 \\
\hline Mimema venturae / 1994 & 6 \\
\hline Phakopsora bluteri / 1993 & 3 \\
\hline Phakopsora chavesi / 1993 & 3 \\
\hline Phakopsora rossmanii / 1993 & 3 \\
\hline Ravenellia bezerrae / 993 & 2 \\
\hline Ravenellia cerradensis / 2001 & 7 \\
\hline Ravenellia chapadensis / 2001 & 7 \\
\hline Ravenellia emaensis / 2001 & 7 \\
\hline Ravenellia mineirosensis / 2001 & 7 \\
\hline Ravenellia santos-costae / 1993 & 2 \\
\hline Ravenellia victoria -rossetii / 1993 & 2 \\
\hline Skierka divinopolenis / 1993 & 2 \\
\hline Uromyces galactiae / 2003 & 8 \\
\hline Uromyces hawksworthii / 2015 & 9 \\
\hline
\end{tabular}

*Type-species of new genera. ${ }^{1}$ Dianese \& Santos (1995). ${ }^{3}$ Dianese et al. (1993d). ${ }^{4}$ Dianese et al. (1993a). ${ }^{5}$ Dianese et al. (1994a). ${ }^{7}$ Dianese et al. (1995). ${ }^{8}$ Dianese et al. (1994d). ${ }^{21}$ Rezende \& Dianese (2001).

${ }^{22}$ Rezende \& Dianese (2003). ${ }^{45}$ Souza et al. (2015). ${ }^{48}$ Souza et al. (2018). thrix usneoides, Crossopsora hymenaeae, Kimuromyces cerradensis, Maravalia perae, Phakopsora coca, Phakopsora rossmaniae, Porotenus bibasiporulus, Prospodium destruens, Pr. fimbriatum, Puccinia banisteriae, P. calida, P. faceta, P. paranahybae, $P$. subcoronata, $P$. uleana, Ravenelia dieteliana, $R$. emaensis, $R$. goyazensis, $R$. mineirosensis, $R$. spiralis, $R$. uleana, Uredo dalbergiae, U. longipedis, U. pithecolobii, U. psychotriicola, Uromyces cnidoscoli, Um. dichorisandrae, Um. goyazensis, Um hawksworhii (= A. goyazense), Um. manihoticola, Um. manihotis); Distrito Federal (Cerotelium xylopiae, Cerradoa palmaea, Crossopsora asclepidiaceae, Mimema venturae, Phakopsora butleri, Ph. chavesii, Ph. tocoyenae. Ravenelia santos-costae, Uromyces galactiae); Mato Grosso (Aecidium calosporum, A. mattogrossense, A. momordicae, Crossopsora angusta, Leptinia brasiliensis, Puccinia callosa, Ravenelia cerradensis, Uromyces anthemophilus, Um. foveolatus); Mato Grosso do Sul (Cerotelium coccolobae, Dicheirinia antunesii, Prospodium scitulum, Ravenelia pernigra); Minas Gerais (Aecidium alternantherae, A. diospyri-hispidae, A. guatteriae, A. minimum, Aplopsora hennenii, Batistopsora crucis-filii, Catenulopsora henneneae, Cerotelium giacomettii, Chaconia maprouneae, Didymopsora triumfettae, Dietelia duguetiae, Diorchidiella verlandii, Phakopsora colubrinae, Porotenus concavus, Prospodium arrabidaeae, Pr. bicolor, Pr. cremastum, Pr. funalis, Pr. pseudozonatum, Puccinia cavatica, $P$. circinans, $P$. lisianthi, $P$.

Table 3. Number of records per taxon, and sites where the rust fungi on native plants from the Cerrado were collected and deposited in the Mycological Collection of Herbarium UB - MCHUB.

\begin{tabular}{lcc}
\hline TAXON & RECORDS & COLLCTION SITES** \\
Aecidium jacarandae & 2 & others* \\
Aecidium sp. & 12 & 1,16, others \\
Aecidium ulei & 78 & $9,11,12,13,17$, others \\
Aplopsora hennenii & 19 & $9,11,13$, others \\
Austropuccinia psidii & 82 & $11,12,13,17$, others \\
Batistopsora crucis-filii & 54 & $3,9,11,17$, others \\
Caeoma sp. & 1 & others \\
Catenulopsora henneneae & 44 & $3,13,17$, others \\
Cerotelium fici & 1 & Others \\
Cerotelium giacometti & 19 & 9,17, others \\
Cerotelium malvicolum & 1 & others \\
Cerotelium sabiceae & 15 & $5,9,17$, others \\
Cerotelium sp. & 1 & others \\
Cerradoa palmaea & 59 & $3,5,9,11,17,19$, others
\end{tabular}


Table 3. Continued

\begin{tabular}{|c|c|c|}
\hline TAXON & RECORDS & COLLECTION SITES** \\
\hline Chaconia apicrassa & 1 & 7 \\
\hline Chaconia brasiliensis & 103 & $2,9,11,13,14,16,17$, others \\
\hline Chaconia ingae & 12 & 16 , others \\
\hline Chaconia maprouneae & 64 & $9,11,12,13,17,18,19$, others \\
\hline Chaconia sp. & 1 & others \\
\hline Cionothrix praelonga & 17 & others \\
\hline Coeoma sp. & 1 & 13 \\
\hline Coleosporium elephantopodis & 4 & others \\
\hline Coleosporium ipomoeae & 5 & 9 , others \\
\hline Coleosporium plumeriae & 6 & others \\
\hline Coleosporium vernoniae & 17 & $16,17,18$, others \\
\hline Crossopsorella byrsonimae & 40 & $6,11,17,18$, others \\
\hline Crossopsora hymenaeae & 60 & $2,9,11,12,13,17$, others \\
\hline Crossopsora sp. & 92 & $8,13,15,17$, others \\
\hline Crossopsorella sp. & 2 & others \\
\hline Dasyspora gregária & 81 & $4,6,9,11,16,17$, others \\
\hline Dasyspora sp. & 1 & others \\
\hline Desmella aneimiae & 2 & others \\
\hline Dietelia duguetiae & 30 & $9,13,15,17$, others \\
\hline Diorchidium copaiferae & 20 & 12 , others \\
\hline Diorchidium sp. & 1 & 16 \\
\hline Esalque holwayi & 2 & others \\
\hline Hemileia vastatrix & 4 & others \\
\hline Kernkampella appendiculata & 5 & others \\
\hline Kimuromyces cerradensis & 27 & others \\
\hline Kweilingia divina & 2 & others \\
\hline Maravalaia erythroxyli & 4 & 13 , others \\
\hline Maravalia bauhinicola & 5 & others \\
\hline Maravalia erythroxyli & 44 & $11,12,15,17$, others \\
\hline Melampsora medusae & 3 & others \\
\hline Melampsora salix & 1 & others \\
\hline Mimema venturae & 24 & 12,15 , others \\
\hline Olivea tectonae & 4 & others \\
\hline Olivea viticis & 3 & others \\
\hline Phakopsora arthuriana & 1 & others \\
\hline Phakopsora butleri & 36 & $3,9,10,12,13,17$, others \\
\hline Phakopsora cf. pávida & 5 & $9,13,17$, others \\
\hline Phakopsora chavesii & 42 & 4,13, others \\
\hline Phakopsora colubrinae & 1 & others \\
\hline Phakopsora crotalariae & 1 & others \\
\hline Phakopsora erythroxyli & 1 & others \\
\hline Phakopsora euvites & 1 & others \\
\hline Phakopsora gossypii & 4 & others \\
\hline Phakopsora mori & 2 & others \\
\hline
\end{tabular}


Table 3. Continued

\section{TAXON}

Phakopsora pachyrhizi

Phakopsora rossmaniae

Phakopsora sp.

Physopella tocoyenae

Porotenus concavus

Porotenus sp.

Prospodium appendiculatum

Prospodium bicolor

Prospodium impolitum

Prospodium pseudozonatum

Prospodium sp.

Prospodium tecomicola

Puccinia acanthospermi

Puccinia andropogonis

Puccinia arechavaletae

Puccinia aristae

Puccinia aspiliae

Puccinia baccharidis

Puccinia banistariae

Puccinia barbatula

Puccinia caxiuanensis

Puccinia cf versicolor

Puccinia cf. calosa

Puccinia cf. mandevillae

Puccinia cf. neorotundata

Puccinia cnici-oleracei

Puccinia conyzae

Puccinia cordiae

Puccinia coronata

Puccinia crepidis

Puccinia elongata

Puccinia farameae

Puccinia gibertii

Puccinia graminis

Puccinia heterospora

Puccinia inrecta

Puccinia lantanae

Puccinia lateritia

Puccinia leonotidis

Puccinia levis

Puccinia melanocephala

Puccinia mikaniae

Puccinia mogiphanis

Puccinia neorotundata

\section{RECORDS}

6

20

9

1

20

1

1

1

1

1

13

1

1

7

28

1

4

10

77

64

1

1

1

1

2

2

1

2

2

1

3

5

6

2

7

2

2

2

1

7

1

2

1

2

\section{COLLECTION SITES**}

others

13 , others

3 , others

3

11,17 , others

others

others

17

others

others

17 , others

others

others

12,16 others

$12,14,17$, others

others

others

13 , others

$9,11,12,15,17$, others

$9,10,11,13,17$, others

others

others

13

1

others

others

others

12 , others

others

others

others

$4,12,14,15$, others

15 , others

16 , others

11 , others

1,17

17 , others

others

others

others

others

others

others 
Table 3. Continued

\section{TAXON}

Puccinia niederleini

Puccinia oxalidis

Puccinia palicourea

Puccinia pelargonii-zonalis

Puccinia pipta

Puccinia psychotriae

Puccinia purpurea

Puccinia recondita

Puccinia rubigo-vera

Puccinia rugose

Puccinia sherardiana

Puccinia smilacis

Puccinia sorghi

Puccinia sp.

Puccinia substriata

Puccinia thaliae

Pucciniaceae non identified

Pucciniales non identified

Pucciniosira pallidula

Pucinia cnici-oleracei

Ravenelia bezerrae

Ravenelia cerradensis

Ravenelia chapadensis

Ravenelia densifera

Ravenelia dieteliana

Ravenelia emaensis

Ravenelia geminipora

Ravenelia goyazensis

Ravenelia hieronymi

Ravenelia lonchocarpi

Ravenelia mimosae-sensitivae

Ravenelia mineiroensis

Ravenelia pernigra

Ravenelia pileolarioides

Ravenelia santos-costae

Ravenelia sp.

Ravenelia sp. nov.

Skierka divinopolensis

Sphaerophragmium acaciae

Sphenospora smilacina

Tranzschelia discolor

Tranzschelia pruni-spinosae

Tranzschelia sp.

Uredo borreriae

\section{RECORDS}

4

4

33

3

60

1

4

1

1

3

1

1

2

32

1

5

2

298

1

1

20

32

17

5

4

17

53

2

6

2

17

1

2

1

70

32

1

31

7

2

1

5

1

1

\section{COLLECTION SITES**}

others

13 , others

others

others

$9,11,12,13,17$, others

9

others

others

others

12 , others

others

others

13 , others

13,17 , others

others

others

others

others

others

17 , others

$5,9,17$, others

$3,4,5,17$, others

others

4,17, others

3 , others

$2,11,16,17$, others

others

others

others

3,17 , others

3

others

others

$9,11,13,15,17$, others

$3,13,16,17$, others

others

$2,9,16$, others

others

9, others

others

others

others

others 
Table 3. Continued

\begin{tabular}{|c|c|c|}
\hline TAXON & RECORDS & COLLECTION SITES** \\
\hline Uredo eriosemae & 1 & 11 \\
\hline Uredo jatrophicola & 1 & others \\
\hline Uredo lafvdensiae & 11 & others \\
\hline Uredo mogy-mirim & 1 & others \\
\hline Uredo sp. & 39 & $11,13,17$, others \\
\hline Uredopeltis guettardae & 5 & 1 \\
\hline Uromyces appendiculatus & 5 & 13 , others \\
\hline Uromyces bauhineae & 48 & $4,9,11,15,17$, others \\
\hline Uromyces bauhinicola & 1 & others \\
\hline Uromyces bidenticola & 1 & others \\
\hline Uromyces bradburyae & 1 & others \\
\hline Uromyces cestri & 1 & others \\
\hline Uromyces costaricensis & 1 & others \\
\hline Uromyces dolicholii & 1 & others \\
\hline Uromyces eriosemae & 3 & 9 , others \\
\hline Uromyces euphorbiae & 4 & 13 , others \\
\hline Uromyces floralis & 4 & others \\
\hline Uromyces foveolatus & 2 & others \\
\hline Uromyces galactiae & 4 & 17 , others \\
\hline Uromyces goyazensis & 13 & $9,12,13$, others \\
\hline Uromyces hawksworthii & 52 & $9,11,13,17$, others \\
\hline Uromyces hemmendorffii & 1 & others \\
\hline Uromyces ipatingae & 1 & others \\
\hline Uromyces manihotis & 4 & others \\
\hline Uromyces mikaniae & 3 & others \\
\hline Uromyces myrsines & 1 & others \\
\hline Uromyces neurocarpi & 12 & 17 , others \\
\hline Uromyces perlebiae & 1 & others \\
\hline Uromyces scleriae & 2 & others \\
\hline Uromyces setatiae-italicae & 3 & others \\
\hline Uromyces sp. & 8 & 1,6 , others \\
\hline Uromyces tolerandus & 1 & others \\
\hline Uromyces transversalis & 5 & others \\
\hline Uromyces viegasii & 7 & others \\
\hline Uromyces vignae & 1 & others \\
\hline
\end{tabular}

others ${ }^{*}=$ Collections in private properties, not from protected sites. ** Oficial Cerrado Reserves:1-Chapada dos Guimarães National Park, 2-Serra do Cipó National Park, 3-Emas National Park, 4-Grande Sertão Veredas National Park, 5-Chapada dos Veadeiros National Park, 6-Chapada das Mesas National Park, 7-Serra da Bodoquena National Park, 8-Sete Cidades National Park, 9-Brasília National Park, 10-Pantanal Matogrossense National Park, 11-Brasilia Botanic Garden, 12-Brasília IBGE Reserve, 13-Brasília Fazenda Água Limpa, 14-Panga-Uberlândia Reserve, 15-Embrapa Reserve, Campo Grande, 16-Embrapa Reserve, Brasília, 17-Águas Emendadas Ecological Station, 18-Serra de Ricardo Franco State Park, 19-Serra Azul State Park, 20- Mogi Mirim Experimental Station, 21-Luis Antônio Experimental Station, 22- Mogi Guaçu Experimental Station.

opulentissima, P. velata, Ravenelia geminipora, $R$. lata, R. lonchocarpi, R. septata, Skierka divinopolensis, Uredo bauhiniae, U. cassiae-rugosae, U. hyptidis, Uromyces. brasiliensis, Um. loranthi, Um. myrsines); Roraima (Ravenelia armata); São Paulo (Cerotelium figueiredeae, Chaconia brasiliensis, Crossopsora byrsonimae, Diorchidium copaiferae, Maravalia erythroxyli (= Puccinia erythroxyli), Neopuccinia bursa, Prospodium impolitum, Pr. palmatum, Puccinia achyroclines, $P$. guassuensis, $P$. manuelensis, $P$. seor- 
sa, P. valentula, P. vinulla, Ravenelia dentifera, $R$. microspora, Uredo mogy-mirim, Uromyces vicinus).

Among the valid names mentioned, 32 have the authorship connected with Prof. Joe F. Hennen, retired from the Arthur Fungarium at Purdue University, now connected with the Botanical Research Institute-Texas. He is an outstanding expert in rust fungi from the Cerrado, where he successfully collected for over 22 years. As registered in Fungarium IBI-Instituto Biológico de São Paulo, his first collection in the Cerrado was on September 02, 1976 (Accession number: IBI-12441) when he and Y. Ono described Cerradoa palmaea in Brasilia at the Estação Ecológica de Águas Emendadas. His last record at IBI dates June 12, 1999 (Accession number: IBI18914), in a Cerrado protected area at Mogi Guaçu, State of São Paulo (FIGUEIREDO \& HENNEN 1998, CARVALHO-JUNIOR et al. 2008). Hennen topped his activities in Brazil with a monumental contribution, the publication of a 490-page review of the Brazilian Pucciniales that became the main repository of information for anybody dealing with Brazilian rust fungi (HENNEN et al. 2005).

At MCHUB, for the first-time rust fungi were described on two important plant families from the Cerrado: Caryocaraceae, Cerotelium giacomettii, on Caryocar brasiliense; and Aplopsora hennenii on Qualea multiflora, a member of the Vochysiaceae (DIANESE et al. 1993d, DIANESE \& SANTOS 1995). A second species of the rare genus Skierka (S. divinopolensis) was described on Matayba guianensis (Sapindaceae) from the Cerrado (DIANESE et al. 1993d, HENNEN et al. 2005). Another highlight was the complete description of the first Uromyces species (U. hawksworthii) from the Cerrado infecting a plant hemiparasite, Phthirusa stelis (Lotanthaceae) (SOUZA et al. 2015), previously described in its asexual form (Aecidium goyazense) by Hennings (1895).

Dianese et al. (1993d), surveying the rust fungi on Fabaceae, described initially two new $R a$ venelia species ( $R$. bezerrae, and $R$. santos-costae), and later $R$. cerradensis, $R$. chapadensis, $R$. emaensis, and $R$. mineirosensis (Rezende and Dianese 2001, 2003b). Presently, with six Ravenelia species published, the contribution by MCHUB comprises $c a$ $15 \%$ of the species known in Brazil (HENNEN et al. 2005). A set of three more Ravenelia species is being processed, including a new genus in family Raveneliaceae.

Cerradoa is the first genus of rust fungi from the Cerrado and first Pucciniales with sexual and asexual morphs present on a palm tree. However, the host was originally misidentified as Attalea gearaensis (HENNEN \& ONO 1978), but with the reexamination of the holotype, PUR F18664, it became clear that the real host is Syagrus comosa, as well as a preliminary phylogenetic study allocated the genus in family Pucciniaceae (GALVÃO-ELIAS et al. 2018). The detailed phylogeny of the genus has been submitted for publication and is in final review by Mycologia. A second host species of $C$. palmaea (S. flexuosa) is long known from the Cerrado (DIANESE et al. 1992).

Dianese et al. (1994a) described a new Crossopsora species (C. hymenaeae) on Hymenaea stigonocarpa, the first Crossopsora on Fabaceae in the Cerrado. However, the Crossopsora species most frequently found in this biome is $C$. byrsonimae infecting Byrsonima species (Malpighiaceae). Surprisingly, recent review including molecular phylogeny showed that this species does not belong in Crossopsora. It belongs in the new genus Crossopsorella, type species Crossopsorella byrsonimae (SOUZA et al. 2018), the fourth genus from the Cerrado, after Porotenus (VIÉGAS 1960), Batistopsora (DIANESE et al. 1993d) and Kimuromyces (DIANESE et al. 1995). A fifth genus, Neopuccinia (MARTINS-JUNIOR et al. 2019), completes the set of cerradoan rust genera.

Carvalho-Junior et al. (2008) in a detailed assessment (from 1975 until 1999), covering three Cerrado reserves (Mogi Mirim, Mogi Guaçu, and Luís Antonio), controlled by the State of São Paulo, Brazil, collected 1,176 specimens, and identified 157 species of Pucciniales on native Cerrado plants. The majority (120 species) dispersed among the genera Puccinia (53), Uromyces (19), Aecidium (10), Phakopsora (10), Prospodium (8), Uredo (6), Crossopsora (5), Ravenelia (5) and Coleosporium (4). The hosts belonged in 128 genera, distributed in 49 botanical families. The taxonomical novelties for the State of São Paulo were published later (CARVALHO-JUNIOR \& HENNEN 2009, 2010, 2012, 2018. 2019, MARTINS-JUNIOR et al. 2019), that included Puccinia manuelensis, P. guassuensis and Neopuccinia bursa. In addition, Carvalho-Junior \& Hennen (2008) described four new species of rust fungi from Goiás (Mavavalia perae, Prospodium destruens, Pr. fimbriatum, Puccinia calida), two from Minas Gerais (Prospodium funalis, Puccinia opulentíssima, and Dicheirinia antunesii from Mato Grosso do Sul. 
Finally, to demonstrate the entire collection effort developed in the Cerrado by the MCHUB and $\mathrm{IBI}$, the map in Figure 3 expresses the extension and intensity of field sampling directed to rust fungi, covering the critical sites within the entire Cerrado biome.

\section{STUDIES ON ASCOMYCETES}

\section{Ascomycetes asexual morphs}

Contributions of the MCHUB to the study of Cerrado asexual ascomycetes were summarized in the Table 4. The first species ever described in the
MCHUB was Phloeosporella flavio-moralis on Eugenia lutescens (DIANESE et al. 1993b), followed by Coniella costae on Myrcia tomentosa (DIANESE et al. 1993a), and P. kytajimae on E. dysenterica (DIANESE et al. 1993c).

The cercosporoid fungi remains as the main group studied at the MCHUB with a total of 77 introduced taxa, distributed in genera Asperisporium, Cercospora, Mycovellosiella (=Passalora p.p.), Passalora, Phaeoramularia (=Passalora), Prathigada (=Passalora), Pseudocercospora, Sirosporium, and Zasmidium (=Stenella). Among them Pseudocercos-

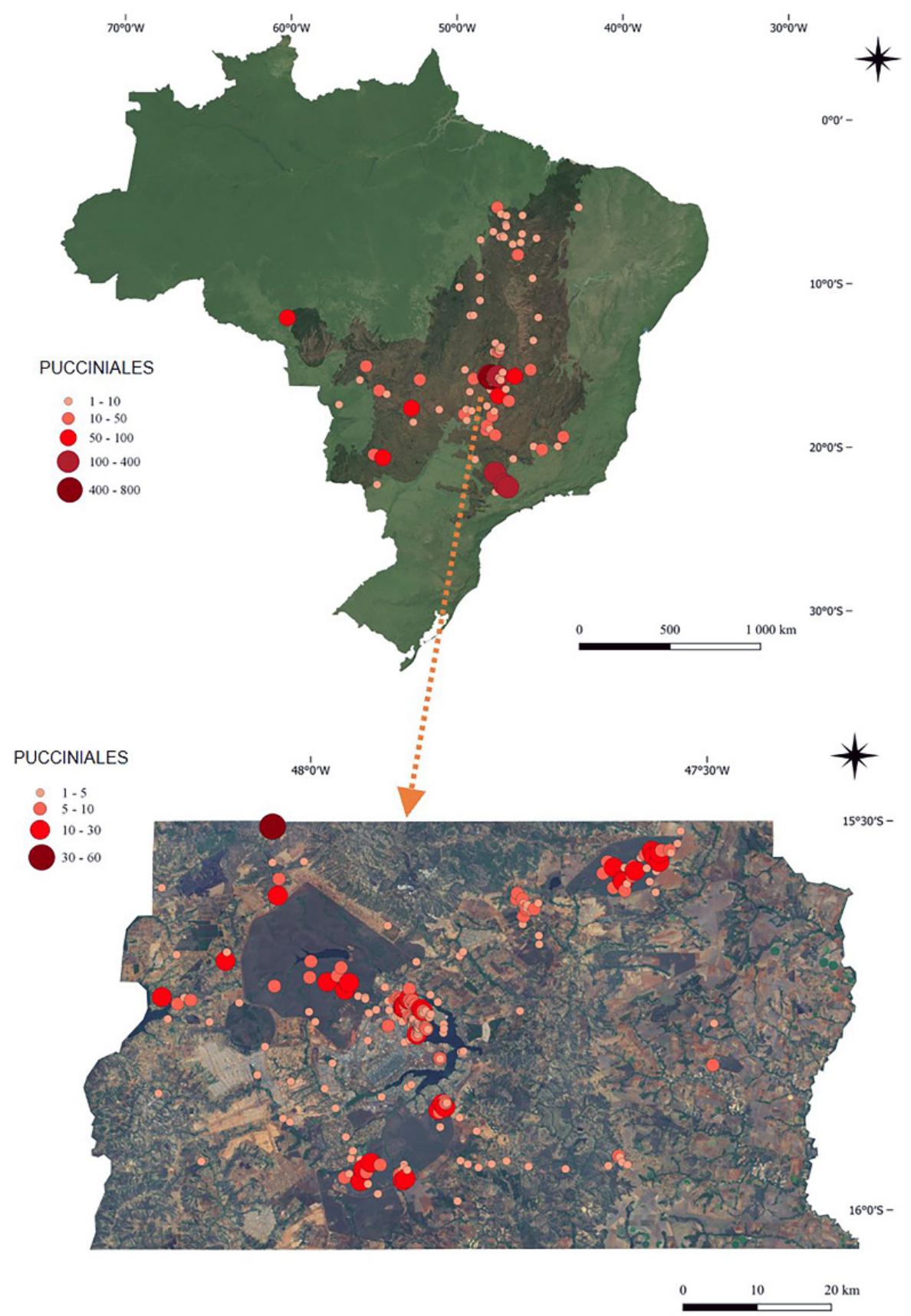

Figure 3. Collection sites in the Brazilian Cerrado (top map), and a detailed view of the activity in the Federal District (bottom), related to the 2,603 specimens of Pucciniales incorporated into the MCHUB starting in 1993. Diameters of the circles are proportional to the number of specimens collected in each site. 
pora is the most important genus with $55 \%$ of the cercosporoid fungi described, followed by Passalora, and Cercospora (Table 4) (DIANESE et al. 1999; DIANESE et al. 2008; DIANESE et al. 2014; DORNELO-SILVA \& DIANESE 2003; DORNELO-SILVA et al. 2007; FURLANETTO \& DIANESE 1999; HERNÁNDEZ-GUTIÉRREZ \& DIANESE 2008, 2009, 2014ab; HERNÁNDEZ-GUTIÉRREZ et al. 2014, 2015; INÁCIO \& DIANESE 1998).

Another set of species that deserve special recognition are the eight new genera of trichomatous hyphomycetes found on members of the Icacinaceae, Malphigiaceae, Fabaceae, Dilleniaceae, Chrysobalanaceae, and Caryocaraceae, revealing the trichomes as an unusual site of epiphyllous fungal diversity (PEREIRA-CARVALHO et al. 2009a), sometimes with parasitic ability (CANTRELL et al. 2011). An ongoing contribution reveals that a fungus hyperparasite of a Phyllachora species, in leaves of Eugenia florida from the Cerrado, treated as a basidiomycete for 134 years since its original description by Spegazzini (1886) as Microcera clavariella, and named Clavaria parasitica Viégas \& Texeira (VIÉGAS \& TEIXEIRA 1945), was shown now to be an ascomycete, Cladosterigma clavariellum, inserted in Gomphillaceae (Lecanoromycetes, Graphidales), a family of predominantly lichenized fungi (SANTOS et al. 2020).

Contribution of the HCHUB to the taxonomy of the asexual ascomycetes was included in the Table 4 .

Table 4. Ascomycete asexual morphs with holotypes in the Mycological Collection of Herbarium UB, starting on 1993, showing 105 new taxa, with 12 type species of new genera.

\begin{tabular}{ll}
\hline \multicolumn{1}{c}{ Species / Year } & Source \\
\hline Alternaria qualeae / 2003 & $\mathbf{1}$ \\
\hline Asperisporium galactiae / 2015 & $\mathbf{2}$ \\
\hline Cercospora jatrophiphila / 2014 & $\mathbf{3}$ \\
\hline Cercospora mimosae-sensitivae / 2009 & $\mathbf{4}$ \\
\hline Cercospora ochromae / 2008 & $\mathbf{5}$ \\
\hline Cercospora tabebuia-impetiginosae / 1998 & $\mathbf{6}$ \\
\hline Coniella costae / 1993 & $\mathbf{7}$ \\
\hline Dinemasporium duguetiae / 1998 & $\mathbf{8}$ \\
\hline Echinoconidiophorum cerradense / 2009* & $\mathbf{9}$ \\
\hline Fumagospora tabebuiae / 1998 & $\mathbf{6}$ \\
\hline
\end{tabular}

Table 4. Continued

\begin{tabular}{|c|c|}
\hline Species / Year & Source \\
\hline Globoconidiopsis cerradensis / 2009* & 9 \\
\hline Globoconidium cerradense / 2009* & 9 \\
\hline Harknessia qualeae / 1998 & 8 \\
\hline Harknessia salvertiana / 1998 & 8 \\
\hline Helminthosporiomyces cerradensis / 2009* & 9 \\
\hline Janetia salvertia / 2003 & 1 \\
\hline Mycovellosiella micranthae / 1996 & 10 \\
\hline Oidium bauhiniicola / 2006 & 26 \\
\hline Parastenella callisthenis-fasciculatae / 2007 & 12 \\
\hline Passalora acosmii / 2009 & 4 \\
\hline Passalora chamaecristae-orbiculatae / 2009 & 4 \\
\hline Passalora chamaecristicola / 2009 & 4 \\
\hline Passalora chapadensis / 2014 & 13 \\
\hline Passalora dalbergiae / 2009 & 4 \\
\hline Passalora delamonicae / 2014 & 13 \\
\hline Passalora eitenii / 1994 & 14 \\
\hline Passalora guimaranhensis / 2014 & 13 \\
\hline Passalora machaerii / 2009 & 4 \\
\hline $\begin{array}{l}\text { Passalora myracrodruonis / } 2003 \text { [三 Mycovello- } \\
\text { siella myracrodruonis] }\end{array}$ & 11 \\
\hline $\begin{array}{l}\text { Passalora peixotoae / [ } \equiv \text { Mycovellosiella pei- } \\
\text { xotoae] }\end{array}$ & 10 \\
\hline Passalora qualeae / 2003 & 1 \\
\hline Passalora schefflerae / 2008 & 5 \\
\hline Passalora tabebuiae-ochraceae / 2006 & 15 \\
\hline Passalora cerradensis / 2014 & 16 \\
\hline Passalora peixotae-reticulatae / 2014 & 16 \\
\hline Passalora brasilianensis / 2014 & 16 \\
\hline Passalora peixotoae-goianae / 2014 & 16 \\
\hline $\begin{array}{l}\text { Passalora austroplenckiae / } 2013 \text { [三 Prathiga- } \\
\text { da austroplenckiae / 2008] }\end{array}$ & 5 \\
\hline $\begin{array}{l}\text { Passalora backmanii / } 2013 \text { [三 Prathigada ba- } \\
\text { ckmanii / 1998] }\end{array}$ & 21 \\
\hline Passalora caesalpiniae / 2009 & 4 \\
\hline $\begin{array}{l}\text { Passalora chuppii / } 2003 \text { [三 Phaeoramularia } \\
\text { chupii / 1998] }\end{array}$ & 10 \\
\hline $\begin{array}{l}\text { Passalora pyrostegiae [三 Phaeoramularia } \\
\text { pyrostegiae / 1996] }\end{array}$ & 10 \\
\hline Periconiella longispora / 2003 & 1 \\
\hline Periconiella qualeae-grandiflorae / 2003 & 1 \\
\hline Phaeoidiomyces qualeae / 2004* & 17 \\
\hline
\end{tabular}


Table 4. Continued

\begin{tabular}{|c|c|}
\hline Species / Year & Source \\
\hline Phaeoramularia rubida / 1996 & 10 \\
\hline Phaeostilbelloides velloziae / 2015* & 18 \\
\hline Phloeosporella flavio-moralis / 1993 & 19 \\
\hline Phloeosporella kitajimae / 1993 & 20 \\
\hline Phragmoconidium cerradense / 2009* & 9 \\
\hline Phyllosticta xylopiae-sericeae / 1998 & 8 \\
\hline Polychaeton tabebuiae / 1998 & 6 \\
\hline Pseucercospora astroniiphila / 2008 & 5 \\
\hline Pseudocercospora acosmii-subelegantis / 2015 & 2 \\
\hline Pseudocercospora annellidica / 2013 & 23 \\
\hline $\begin{array}{l}\text { Pseudocercospora aquae-emendadasensis / } \\
2015\end{array}$ & 2 \\
\hline Pseudocercospora astronii / 2008 & 5 \\
\hline Pseudocercospora austroplenckiae / 2008 & 5 \\
\hline $\begin{array}{l}\text { Pseudocercospora banisteriopsis-megaphyllae } \\
\text { / } 2014\end{array}$ & 13 \\
\hline Pseudocercospora bonducella / 2009 & 4 \\
\hline $\begin{array}{l}\text { Pseudocercospora byrsonimae-basilobae / } \\
2013\end{array}$ & 23 \\
\hline $\begin{array}{l}\text { Pseudocercospora byrsonimae-coccolobifoliae } \\
\text { / } 2013\end{array}$ & 23 \\
\hline Pseudocercospora byrsonimicola / 2013 & 23 \\
\hline Pseudocercospora byrsonimigena / 2013 & 23 \\
\hline Pseudocercospora caesalpiniicola / 2009 & 4 \\
\hline Pseudocercospora campograndensis / 2013 & 23 \\
\hline Pseudocercospora chamaecristigena / 2009 & 4 \\
\hline Pseudocercospora eriothecae / 2008 & 5 \\
\hline Pseudocercospora exilis / 2009 & 4 \\
\hline Pseudocercospora grajauensis / 2013 & 23 \\
\hline Pseudocercospora luzianiensis / 2009 & 4 \\
\hline Pseudocercospora matogrossoensis / 2014 & 13 \\
\hline $\begin{array}{l}\text { Pseudocercospora mutabiliconidiophorum / } \\
2013\end{array}$ & 22 \\
\hline Pseudocercospora passiflorae-setaceae / 2008 & 25 \\
\hline Pseudocercospora pediformiconidiorum / 2013 & 22 \\
\hline Pseudocercospora planaltinensis / 2013 & 22 \\
\hline Pseudocercospora protii / 2008 & 5 \\
\hline Pseudocercospora pseudobombacis / 2008 & 5 \\
\hline Pseudocercospora sennae / 2015 & 2 \\
\hline Pseudocercospora sennae-rugosae / 2015 & 2 \\
\hline Pseudocercospora stryphnodendri / 2015 & 2 \\
\hline
\end{tabular}

Table 4. Continued

\begin{tabular}{|c|c|}
\hline Species / Year & Source \\
\hline Pseudocercospora subcuticularis / 2015 & 2 \\
\hline Pseudocercospora subhyalina / 2013 & 2 \\
\hline Pseudocercospora tabebuiae-caraibae / 2006 & 15 \\
\hline Pseudocercospora uwebrauniana / 2014 & 13 \\
\hline Pseudocercospora zeyheriae / 1999 & 23 \\
\hline Pseudocercospora aspidospermatis / 1994 & 24 \\
\hline Pseudocercospora bolkanii / 1999 & 22 \\
\hline Pseudocercospora luzardii / 1999 & 22 \\
\hline $\begin{array}{l}\text { Pseudocercospora tabebuiae-roseo-albae / } \\
1998\end{array}$ & 6 \\
\hline Pseudocercospora urenae / 1996 & 10 \\
\hline Septoria tabebuiae-impetiginosae / 1998 & 6 \\
\hline Sirosporium sclerolobii / 2015 & 2 \\
\hline $\begin{array}{l}\text { Zasmidium cyrtopodii } 2014 \text { / [ } \equiv \text { Stenella cyrto- } \\
\text { podii / 2007] }\end{array}$ & 12 \\
\hline Trichomatoclava cerradensis / 2009* & 21 \\
\hline Trichomatomyces byrsonimae / 2004* & 17 \\
\hline Trichomatosphaera cerradensis / 2009* & 9 \\
\hline Trichosporodochium cerradensis / 2004* & 17 \\
\hline Velloziomyces ramosiconidialis / 2015* & 18 \\
\hline Vesiculohyphomyces cerradensis / 2009* & 9 \\
\hline Zamidium ocotei / 2015 & 2 \\
\hline Zasmidium erythroxyli-campestris / 2015 & 2 \\
\hline Zasmidium erythroxylicolum / 2015 & 2 \\
\hline Zasmidium erythroxyli-suberosi / 2015 & 2 \\
\hline Zasmidium sclerolobii / 2015 & 2 \\
\hline
\end{tabular}

*Type-species of new genera. ${ }^{1}$ Dornelo-Silva \& Dianese (2003). ${ }^{2} \mathrm{He}-$ nández-Gutiérrez et al. (2015). ${ }^{3}$ Dianese et al. (2014). ${ }^{4}$ Hernández-Gutiérrez \& Dianese (2009). ${ }^{5}$ Hernández-Gutiérrez \& Dianese (2008). ${ }^{6}$ Inácio \& Dianese (1998). ${ }^{7}$ Dianese et al. (1993a). ${ }^{8}$ Furlanetto \& Dianese (1997). ${ }^{9}$ Pereira-Carvalho et al (2009). ${ }^{10}$ Inácio et al. (1996). ${ }^{11}$ Inácio \& Dianese (1999). ${ }^{12}$ Dornelo-Silva et al. (2007). ${ }^{13}$ Hernández-Gutiérrez et al. (2014b). ${ }^{14}$ Medeiros \& Dianese (1994). ${ }^{15}$ Inácio \& Dianese (2006). ${ }^{16}$ Hernández-Gutiérrez et al. (2014a). ${ }^{17}$ Dornelo-Silva \& Dianese (2004). ${ }^{18} \mathrm{Armando}$ et al. (2015). ${ }^{19}$ Dianese et al. (1993b). ${ }^{20}$ Dianese et al. (1993c). ${ }^{21}$ Furlanetto \& Dianese (1999). ${ }^{22}$ Hernández-Gutiérrez et al. (2014b). ${ }^{23}$ Dianese et al. (1999). ${ }^{24}$ Dianese \& Câmara (1994). ${ }^{25}$ Dianese et al. (2008). ${ }^{26}$ Braun et al. (2006).

As the cercosporoid fungi (Order Capnodiales, Family Mycosphaerellaceae) constitute the main group of asexual ascomycetes described and deposited in the MCHUB, the distribution of this fungal group through the sites in the Cerrado where extensive collection occurred was shown in the Figure 4. 


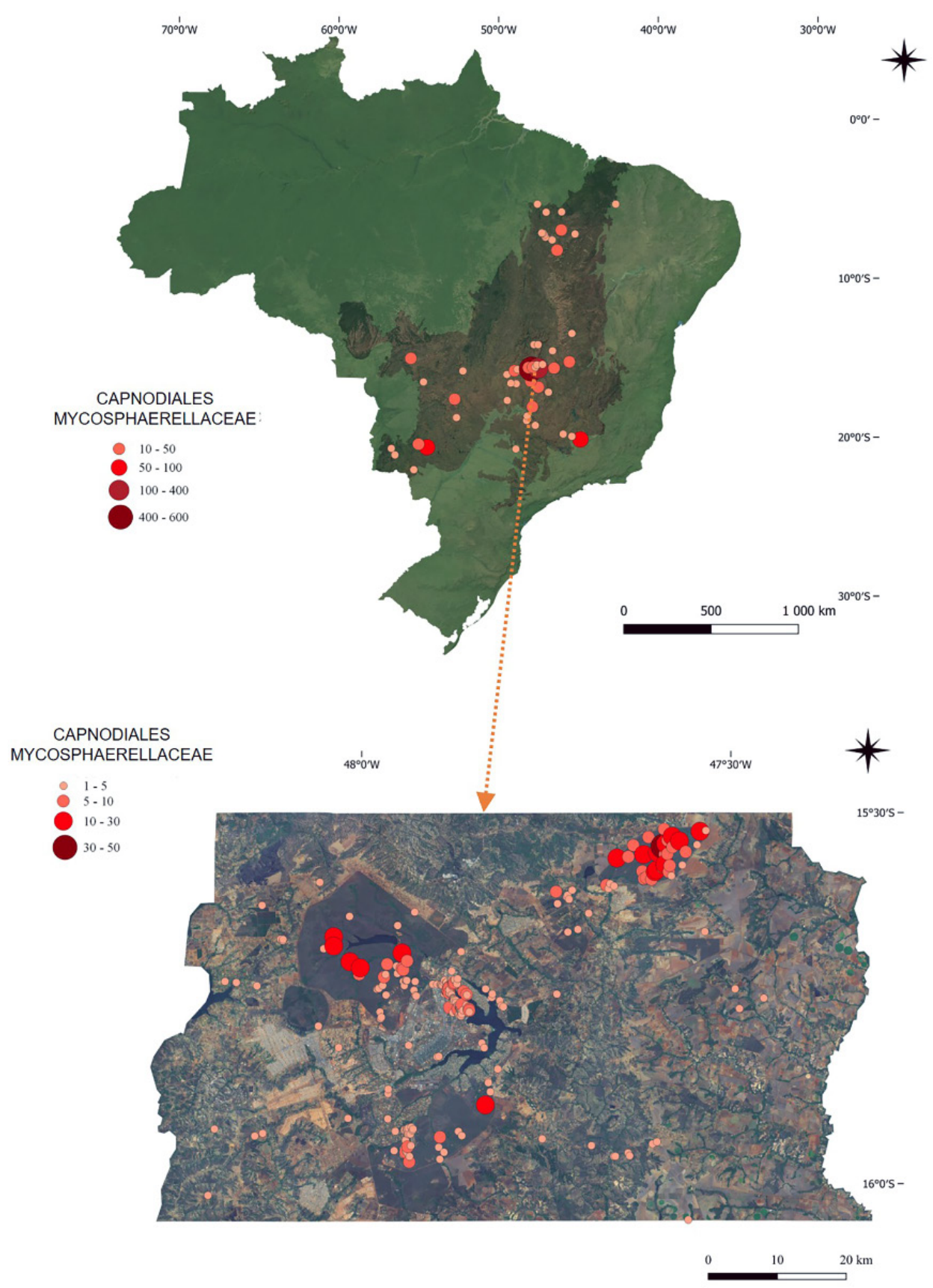

Figure 4. Collection sites in the Brazilian Cerrado (top map), and a detailed view of the activity in the Federal District (bottom), related to the 1,864 specimens of Capnodiales- Mycosphaerellaceae incorporated into the MCHUB starting in 1993. Diameters of the circles are proportional to the number of specimens collected in each site.

\section{STUDIES ON ASCOMYCETE SEXUAL MORPHS}

In Table 5, the main novelties were the introduction of three new genera of ascomycetes, Chaetothyriomyces, Plurispermiopsis, and Wilmia (= Letendraea), and a set of four new Phyllachora species, and two new species in family Parmulariaceae (Dothidasteroma psidii, and Hysterostomella connari). Collection effort related to the three main orders of ascomycetes (Asterinales, Meliolales and Phyllachorales) found in the Cerrado are shown in Figures 5, 6, and 7.

Batista and collaborators also focused the
Cerrado ascomycetes, with most of his contributions included in Silva \& Minter (1995). Specifically dedicated to the Cerrado sexual ascomycetes are Batista \& Peres (1966a), and Batista et al. (1966abcdf, 1967a).

Viégas $(1943,1944,1947)$ and Viégas \& Cardoso (1944), studying a set of Cerrado fungi described the sexual morphs of Brasiliomyces (type species Brasiliomyces malvastri), Capnodium erythrinicola, Catacauma davillae, C. nigerrimum (= Phyllachora nigerrima), C. qualeae Viégas (=Phyllachora qualeae), C. trucatisporum (=Phyllachora truncatis- 
Table 5. Ascomycete sexual morphs described and deposited in the Mycological Collection of Herbarium UB, starting on 1993, containing 27 new taxa described, with three type species of new genera.

\begin{tabular}{|c|c|}
\hline Species / Year & Source \\
\hline Anhelia tabebuiae / 1998 & 1 \\
\hline Asterina cerradensis / 2019 & 2 \\
\hline Asterina malvacearum / 2019 & 2 \\
\hline Lembosia matogrossensis / 2019 & 2 \\
\hline Lembosia miconiphylla / 2019 & 2 \\
\hline Asterolibertia bahiensis / 2016 & 3 \\
\hline Asterolibertia barrinhensis / 2016 & 3 \\
\hline Asterolibertia campograndensis / 2016 & 3 \\
\hline Asterolibertia parinaricola / 2016 & 3 \\
\hline Camarotella brasilienses / 2008 & 4 \\
\hline Chaetothyriomyces brasiliensis / 2009* & 5 \\
\hline Cirsosia splendida var. laevigata / 2016 & 3 \\
\hline Dictyonella tabebuiae / 1998 & 1 \\
\hline Dothidasteroma psidii / 2011 & 5 \\
\hline Hysterostomella connari / 2012 & 6 \\
\hline Meliola albiziae-polyanthae / 2014 & 7 \\
\hline Meliola bodoquensis / 2014 & 7 \\
\hline Meliola sweetiae / 2014 & 7 \\
\hline Meliola andirae-humilis / 2014 & 7 \\
\hline Meliola eriosemae / 2014 & 7 \\
\hline Phyllachora cerradensis / 2016 & 8 \\
\hline Phyllachora ermidensis / 2016 & 8 \\
\hline Phyllachora furnasensis / 2016 & 8 \\
\hline Phyllachora myrciariae / 2016 & 8 \\
\hline Phyllachora nigerrima / 2016 & 8 \\
\hline Plurispermiopsis cerradensis / 2010* & 9 \\
\hline Uncinula viegasii / 1995 & 10 \\
\hline Uncinulla alvinii / 1995 & 10 \\
\hline Uncinulla heringeriana / 1995 & 10 \\
\hline $\begin{array}{l}\text { Wilmia (= Letendraea Sacc.) brasiliensis / } \\
\text { 2001* }\end{array}$ & 11 \\
\hline
\end{tabular}

*Type-species of new genera published. 'Inácio \& Dianese (1998). ${ }^{2}$ Firmino et al. (2019). ${ }^{3}$ Firmino et al. (2016). ${ }^{4}$ Souza et al. (2008). ${ }^{4} \mathrm{Pe}-$ reira-Carvalho et al. (2009). ${ }^{5}$ Inácio et al. (2011). ${ }^{6}$ Inácio et al. (2012). ${ }^{7}$ Soares \& Dianese (2014). ${ }^{8}$ Santos et al. (2016). ${ }^{9}$ Pereira-Carvalho et al. (2010). ${ }^{10}$ Dianese \& Dianese (1995). ${ }^{11}$ Dianese et al. (2001). pora), Diplochorella indaia, Episphaerella didymopanacis, E. myrciae, Elsinoe tecomae, Gaillardiella caryocana, Lasmenia flavozonata, Nectria erythroxylifoliae (=Trichonectria erythroxylifoliae), Perisporina roupalae, Phyllachora myrciae-rostratae, Queirozia, type species Queirozia turbinata (=Pleochaeta turbinata), and Trabutia pampulhae.

The solid repository represented by the MCHUB and the addition of the phylogenetic species concept allows for a wider and more effective taxonomical work and insertion of taxa in the phylogenetic system internationally adopted. Thus, the genera Apiosphaeria (type species $A$. guaranitica) and Phaeochorella (type species $P$. parinari) were long accommodated in the order Phyllachorales. Recent studies focusing on morphology and molecular phylogeny revealed that both genera belong in Diaporthales (GUTERRES et al. 2018, 2019). In addition, Pheochorella was found to belong in a new family Phaeochorellaceae (GUTERRES et al. 2019). In conclusion, the knowledge generated in the MCHUB, with the new molecular tools, offers the opportunity for advancement of taxonomical work with Cerrado fungi.

International recognition for the work at MCHUB was recently expressed by cover pictures shown in fascicles of Plant Disease (AGRA et al. 2018) showing the symptoms of a false smut on beans, and a cover of a Mycologia fascicle illustrated by pictures of Phaeochorella, the type genus of the new family Phaeochorellaceae (GUTERRES et al. 2019). These were the first such happenings ever for Brazilian mycologists in both journal that belong to the American Phytopathological Society and Mycological Society of America, respectively (Figure 8).

\section{GEOGRAPHICAL REPRESENTATION OF THE CERRADO MYCODIVERSITY IN THE MCHUB}

The collection inventory contains microfungi collected from the Cerrado in Federal District and in the States of Bahia, Goiás, Minas Gerais, Mato Grosso, Mato Grosso do Sul, Tocantins, Rondônia, Maranhão, and Piauí. Through the years the field work within the Federal District was shown to be more intensive than in any of the states because of the availability of local resources and the extension and diversity of the natural reserves within its limits. Presently, there are 14,446 fungal specimens deposited in the MCHUB, collected in the Federal District, 


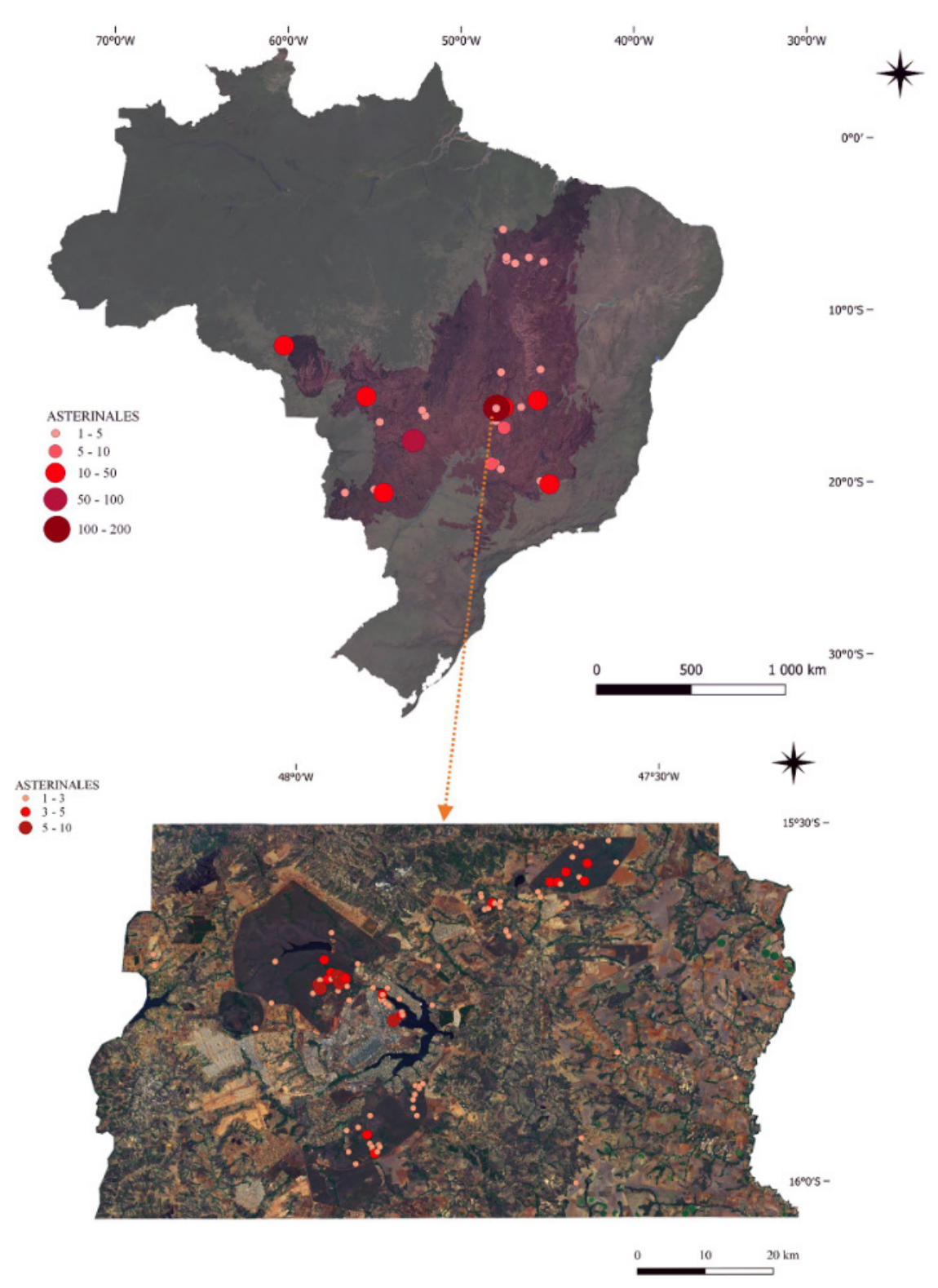

Figure 5. Collection sites in the Brazilian Cerrado (top map), and a detailed view of the activity in the Federal District (bottom), related to 569 specimens of Asterinales- Asterinaceae incorporated into the MCHUB starting in 1993. Diameters of the circles are proportional to the number of specimens collected in each site.

where the major sources of material are: Brasilia National Park, Águas Emendadas Ecological Station, Brasilia Botanical Garden, UnB Fazenda Água Limpa, IBGE Cerrado Reserve, Embrapa Planaltina Cerrado Reserve, Brasilia National Forest, Cafuringa Preservation Area, Cerrado within the 300 hectares UnB Campus, UnB Biological Experiment Station and Arboretum. In Goiás the 2,225 specimens came mainly from two National Parks, Chapada dos Veadeiros in Alto Paraiso and Parque das Emas in Mineiros, Nova India Farm and Vereda do Gato Reserve in Cristalina. In Maranhão, 812 specimens were collected in two different expeditions. During the first expedition the samples were collected along the highways starting in Imperatriz and going to Balsas via Grajaú, concentrating mostly around the Embrapa Experiment Station in Balsas. The round trip involved collecting along the road to Loreto, Extremo, Carolina, and back to Imperatriz. The other field trip was concentrated in the Chapada das Mesas National Park, in Carolina.

The 1,381 specimens from Mato Grosso were distributed among Chapada dos Guimarães National Park, Pantanal National Park, Serra Azul State Park in Barra do Garça, Serra Ricardo Franco State Park in Vila Bela da Santissima Trindade, Serra do Petrovina to Rondonópolis along BR 364 Highway. In the state of Mato Grosso do Sul 1,060 specimens were reco- 


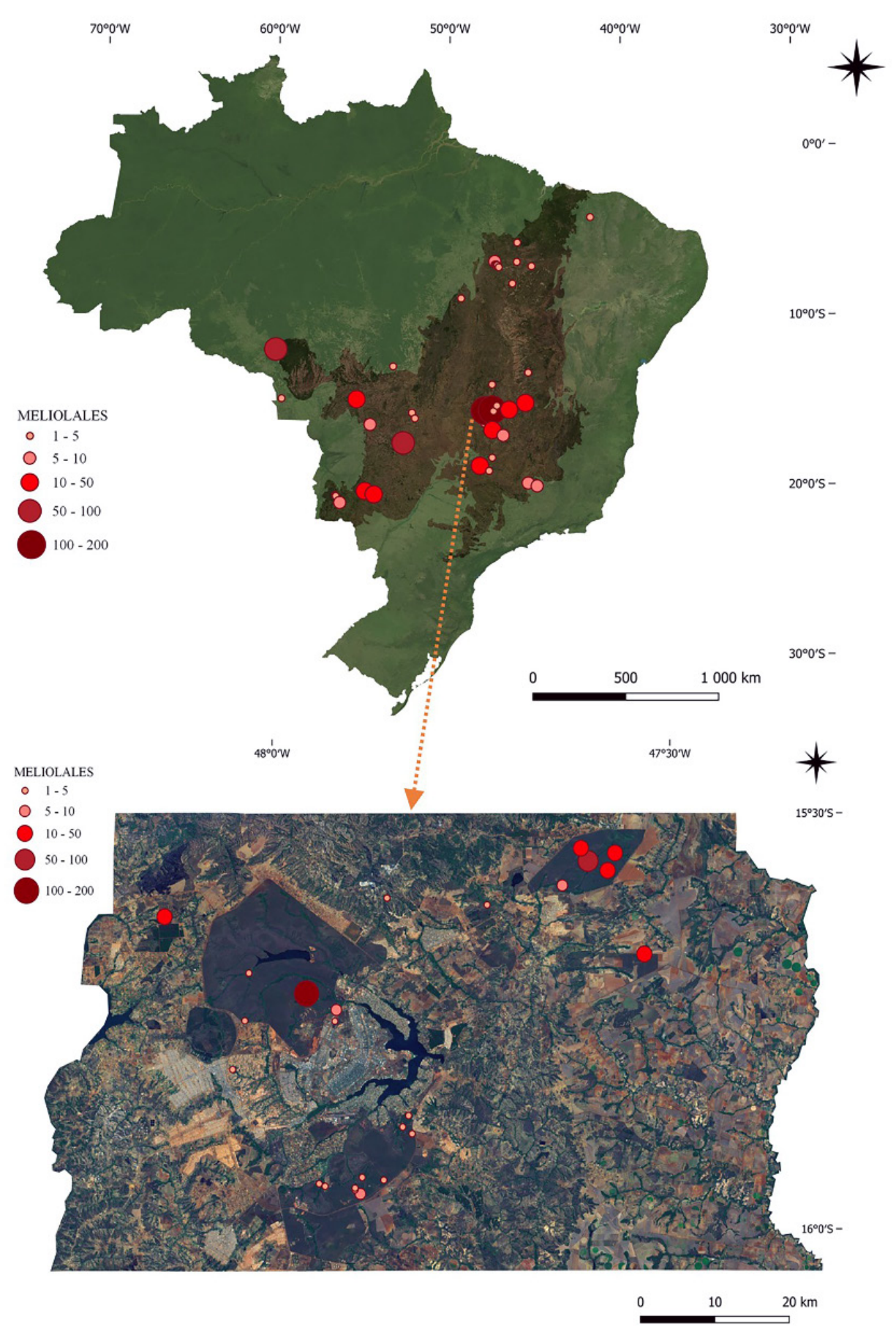

Figure 6. Collection sites in the Brazilian Cerrado (top map), and a detailed view of the activity in the Federal District (bottom), related to the 836 specimens of Meliolales incorporated into the MCHUB starting in 1993. Diameters of the circles are proportional to the number of specimens collected in each site.

vered starting with a large sample taken from the Parque dos Poderes, a permanent Cerrado Reserve, right at the downtown capital Campo Grande, Embrapa Cerrado Reserve at Lagoinha Farm, Cerrados at Terenos (Patagonia Farm and others), several sites at the Pantanal Matogrossense in Aquidauana, Bodoquena, and Miranda.

In Minas Gerais 2,294 specimens were collected covering two National Parks (Grande Sertão Veredas and Serra do Cipó), Panga Ecological Reserve
(Federal University of Uberlândia), Grotadas in Santo Antônio do Monte, Cerrados around Uberlândia, Araguari, Brasília and Prata along the highways BR 050, BR 040 and MG 497, Barrinha Farm (São Sebastião do Oeste), Divinópolis, Marilândia, Pedra Grande Farm (Buritis), Botelho Puntel Farm (Paracatu), around the basins of Furnas, Miranda and Nova Ponte hydroelectric dams.

Only 106 specimens came from Piauí on a trip to the Sete Cidades National Park in Piripiri, going 


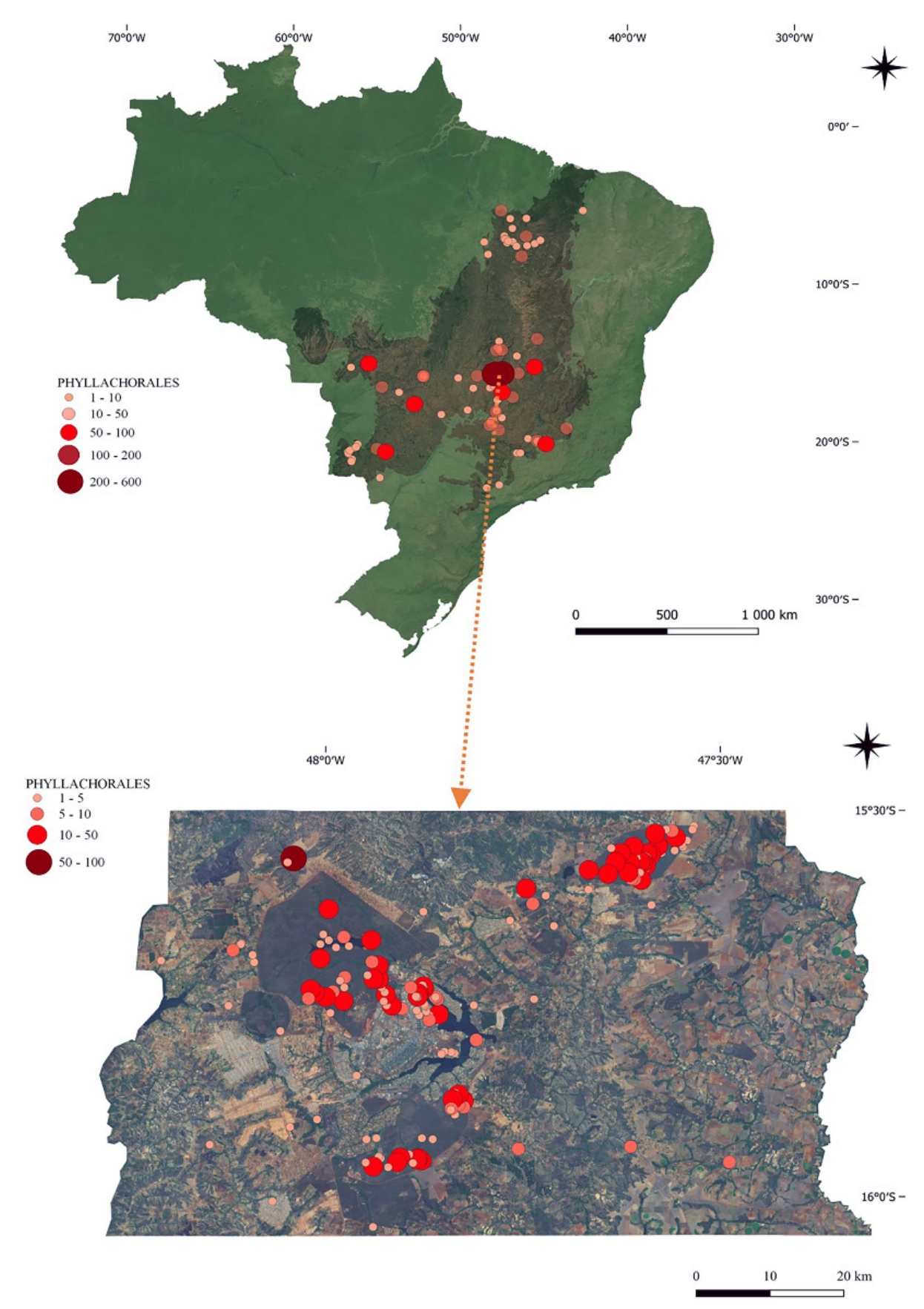

Figure 7. Collection sites in the Brazilian Cerrado (top map), and a detailed view of the activity in the Federal District (bottom), related to the 2501 specimens of Phyllachorales incorporated into the MCHUB starting in 1993. Diameters of the circles are proportional to the number of specimens collected in each site.

through Campo Maior. The State of Tocantins needs to be further surveyed, only 35 specimens from Araguaína, Pedra Branca and Gurupi.

An important historical record is to acknowledge here that over $20 \%$ of the specimens deposited in the MCHUB were collected from over 40 different sites by the late Prof. Mariza Sanchez (Figure 9), who exerted the curatorship of the Collection for over 23 years.

The rust fungi from the State of São Pau- lo were covered in detail by Carvalho-Junior et al. (2008), and their data was included herein. However, concerning other microfungi the Cerrado reserves from Mogi Guaçu, Mogi Mirim and Antônio Alves need to be covered by the MCHUB in the future.

Most plant-associated microfungi from the Cerrado are strictly biotrophic, and the main groups belong to Asterinales, Meliolales, Phyllachorales, and Pucciniales, or highly host specific members of the Capnodiales/ Mycosphaerellaceae (Tables 4 


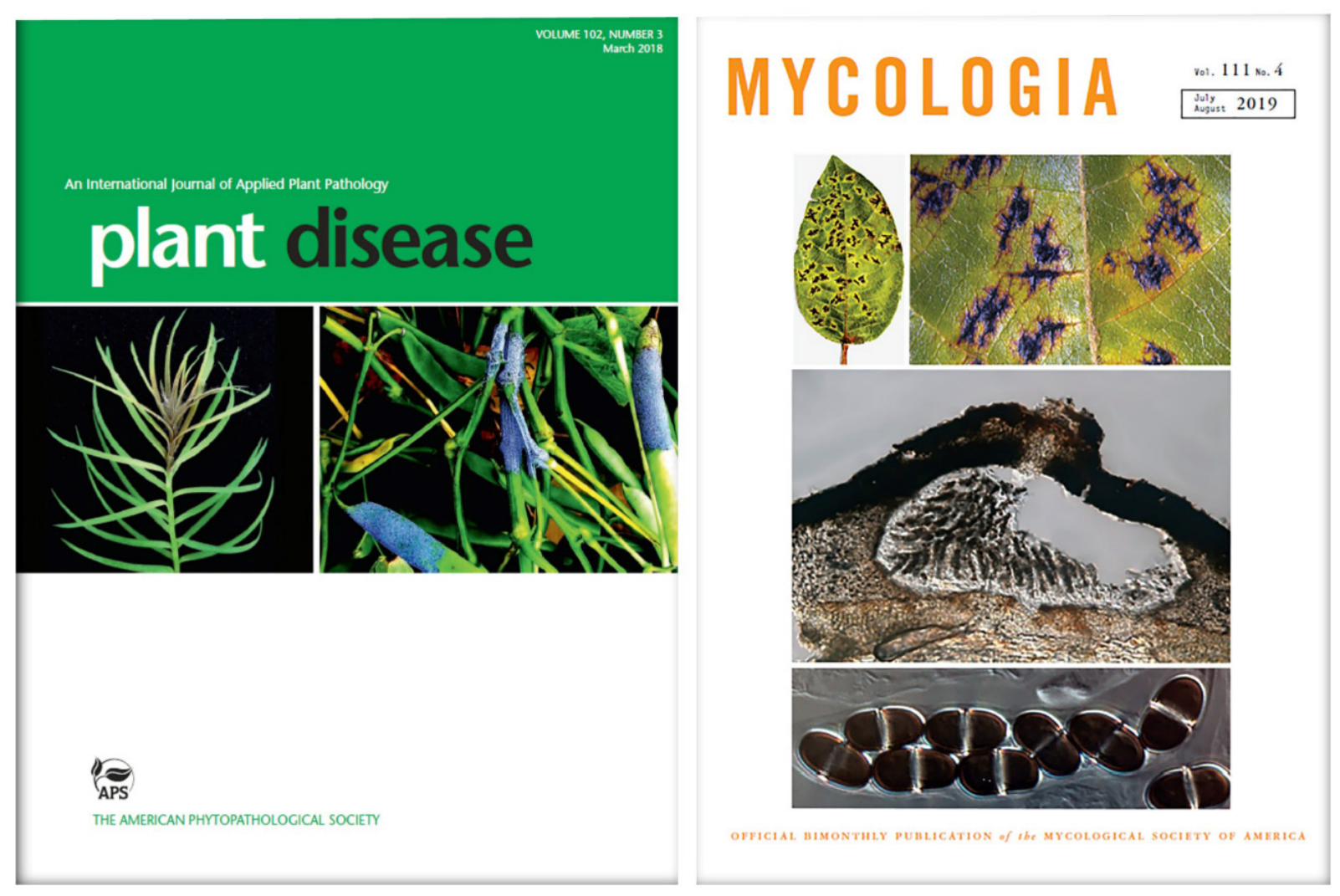

Figure 8. Cover pictures of the journals Plant Disease and Mycologia contributed by Agra et al. (2018) and Guterres et al. (2019) working at the MCHUB, respectively. Pictures included with permission from the American Phytopathological Society and Mycological Society of America.

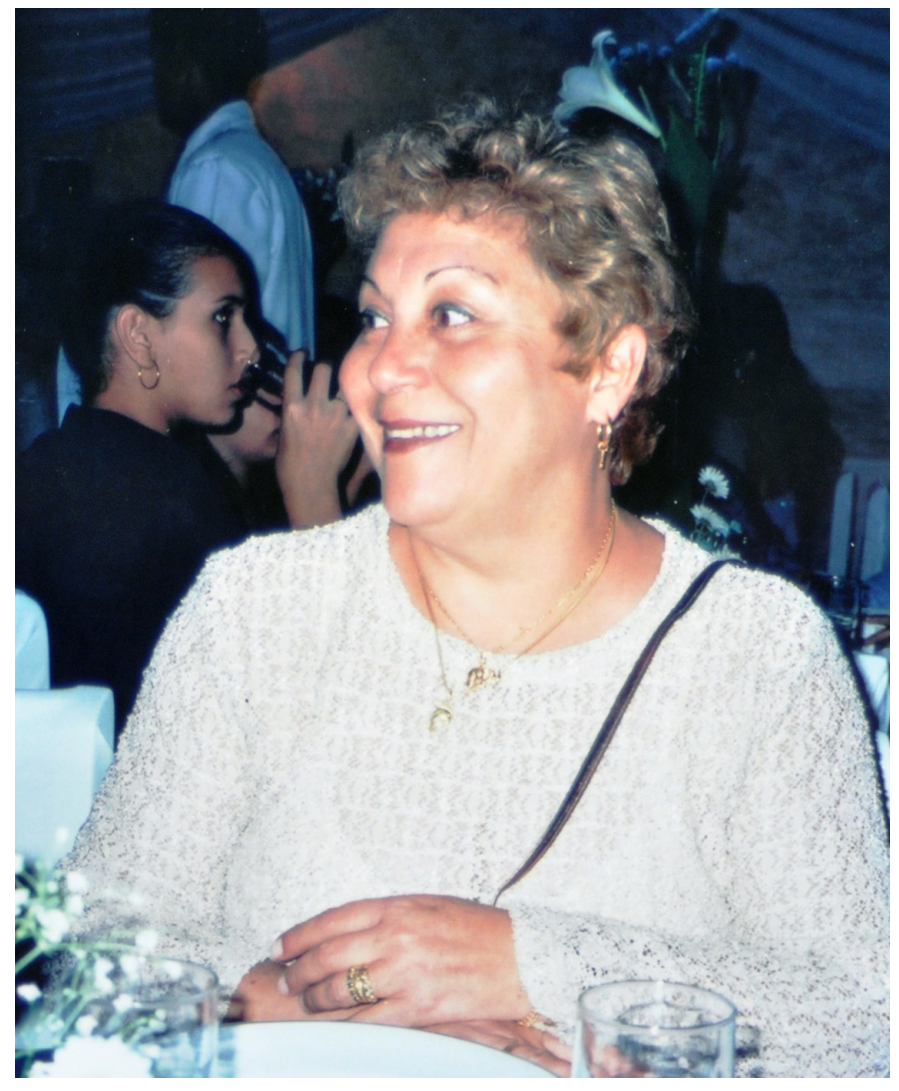

Figure 9. Professor Mariza Sanchez (*1948 -†2015). MCHUB Curator- 1991 to 2014. Photo by Maryland Sanchez - 1999. and 5). Thus, the extinction of rare or endemic plant species occurring in 50 plant families in the Cerrado (MARTINELLI et al. 2014), will also seal the destiny of many microfungal species from the second largest Brazilian biome.

The MCHUB is an internationally recognized collection that contains 148 holotypes, being 18 type species of new genera, needing care on the part of the institution administrative agents to remain as a safe repository of the Cerrado mycodiversity related to the plant-associated microfungi. Almost half of the MCHUB inventory is still to be studied, clearly showing that it is a dynamic institution where at any time researchers will be able to generate mycological knowledge from well preserved samples, mainly because now DNA extraction of herborized material became routine for most of the biotrophic microfungi.

\section{ACKNOWLEDGMENTS}

We thank the Conselho Nacional de Desenvolvimento Científico e Tecnológico for a research grant to JCD (CNPq \# 457455/2012-8) within the "2012 PPBIO-Cerrado Project". JCD thanks "Fundação Banco do Brasil" for the 1995 grant that allowed 
for the assembly of the Herbarium UB Mycological Collection and provided funds for the nationwide collections throughout the Cerrado Biome mostly in National Parks, from 1995 to1998. More recently it was most welcome a grant from the Fundação de Apoio à Pesquisa do Distrito Federal (FAPDF) through the Project FAP-DF \# 417 00193-077/201941, headed by Prof. Dirceu Macagnan.

\section{REFERENCES}

AGRA LANN, SEIXAS CDS, DIANESE JC (2018). False Bean Smut Caused by Slime mold. Plant Disease 102:507-510. (https://doi.org/10.1094/PDIS-0617-0831-RE).

ARMANDO EAS, CHAVES ZM, DIANESE JC (2015). Phaeostilbelloides and Velloziomyces - new dematiaceous genera from the Brazilian Cerrado. Mycotaxon 130:757-767. (https://doi. org/10.5248/130.757).

ARTHUR JC (1925). The Grass Rusts of South America; Based on the Holway Collections. Proceedings of the American Philosophical Society 64:131223. (https://doi.org/ 10.2307/984193).

BURITICÁ P, HENNEN JF (1980). Pucciniosireae (Uredinales, Pucciniaceae) Flora Neotropica 24:148. (https://doi.org/10.2307/3753909).

BATISTA AC, BEZERRA JL, POROCA DJM (1966a). Espécies novas de Sphaeropsidaceae e Tuberculariaceae de Brasilia, D.F. e de Minas Gerais. Atas Inst. Micol. Univ. Fed. Pernambuco, Recife 3:150167.

BATISTA AC, BEZERRA JL, POROCA DJM, MOURA NR (1966b). Espécies novas e antigas de Ascomycetes epifíticos da flora brasílica. Atas do Instituto de Micologia. Universidade Federal de Pernambuco, Recife 3:122-138.

BATISTA AC, BEZERRA JL, CASTRILLÓN AL (1966c). Akaropeltopsis n. gen. e outros Chaerothyriaceae da Amazônia e do Planalto Central brasileiro. Atas do Instituto de Micologia. Universidade Federal de Pernambuco, Recife 3:44-60).

BATISTA AC, PERES GEP (1964). Alguns fungos Cercospora de Minas Gerais. Anais XIII Congresso Nacional Soc. Bot. Brasil. 374-384.

BATISTA AC, PERES GEP (1965). Alguns fungos Sphaeropsidaceae do planalto central Brasileiro. Rivista di Patologia Vegetale Pavia, Ser. 41:51-59.

BATISTA AC, PERES GEP (1966). Sphaeropsidaceae e Nectrioidaceae de comum ocorrência no Planalto Central Brasileiro. Atas Inst. Micol. Univ.
Fed. Pernambuco, Recife 3:202-216.

BATISTA AC, PERES GEP, BEZERRA JL (1962). Alguns Moniliales dos cerrados de Minas Gerais e de Goiás. Publ. Inst. Micol. Universidade do Recife 343:1-26.

BATISTA AC, PERES GEP, CAVALCANTE WA, HERINGER EP (1966d). Alguns Mycosphaerellaceae novos e antigos do Planalto Central Brasileiro. Atas Inst. Micol. Univ. Fed. Pernambuco, Recife 3:218-237.

BATISTA AC, PERES GEP, MAIA HS (1967a). Alguns Phyllachora dos Cerrados e de outras áreas fisiográficas brasileiras. Atas Inst. Micol. Univ. Fed. Pernambuco, Recife 4:63-81.

BATISTA AC, PERES GEP, POROCA DJM (1966e). Especies novas de Sphaeropsidaceae e Tuberculariaceae de Brasília, D.F. e de Minas Gerais Atas do Instituto de Micologia. Universidade Federal de Pernambuco, Recife 3:150-167.

BATISTA AC, PERES GEP, POROCA DJM (1966f). Sphaerodothis diplothemifolii n.sp. e outros Ascomycetes endofíticos do Planalto Central Brasileiro. Atas Inst. Micol. Univ. Fed. Pernambuco, Recife 3:190-201.

BATISTA AC, POROCA DJM, BEZERRA JL, PERES GEP (1967b). Uma coletânea de coelomycetes estudados no IMUFPe. Atas do Instituto de Micologia. Universidade Federal de Pernambuco, Recife 5:73-85.

BERKELEY MJ (1843). Notices of some Brazilian fungi. London J. Bot. 2:629-643.

BERKELEY MJ (1879). Fungi Brasiliensis in provincia Rio de Janeiro q Clar. Dr. A. Glaziou lecti. Videnskabelige Meddelelserfra dansk Naturhistorisk Forening i Kjøbenhavn, 1879-1880:31-34.

BERKELEY MJ, COOK MC (1877). The fungi of Brazil, including those collected by J.W.H. Trail esq. MA in 1874. J. Linnean Soc. 15:82-86. (https://doi.org/10.1111/j.1095-8339.1876.tb00248.x).

BEZERRA JDP, MACIEL MHC, BEZERRA JL, MAGALHÃES OMC, SOUZA-MOTTA CM (2017). The contribution of Augusto Chaves Batista (1916-1967) to mycology in Brazil. Gaia Scientia 11:250-273. (https://doi.org/10.22478/ufpb. 1981-1268.2017v11n2.27916).

BRAUN U, DELHEY R, DIANESE JC, HOSAGOUDAR VB (2006). Miscellaneous notes on biotrophic micromycetes. Schlechtendalia 14:85-97.

CANTRELL SA, DIANESE JC, FELL J, GUNDE-CIMERMAN N, ZALAR P (2011). Unusual fungal 
niches. Mycologia 103:1161-1174. (https://doi. org/10.3852/11-108).

CARDOSO L, VIÉGAS AP (1945). O estado conidiano de Queirozia. Bragantia 5:793-795. (https://doi. org/10.1590/S0006-87051945001200003).

CARNEGIE AJ (2015) First report of Austropuccinia psidii (myrtle rust) in Eucalyptus plantations in Australia. Plant Dis 99: 161. ( https://doi. org/10.1094/PDIS-09-14-0901-PDN).

CARNEGIE AJ, LIDBETTER JK, WALKER J, HORWOOD MA, TESORIERO L, GLEN M, PRIEST MJ (2010). Uredo rangelii, a taxon in the guava rust complex, newly recorded on Myrtaceae in Australia. Australasian Plant Pathology 39:463-466. (https://doi.org/10.1071/AP10102).

CARNEIRO LS (1968). Augusto Chaves Batista (19051967). Mycologia 60:1137-1139. (https://doi.org /10.1080/00275514.1968.12018680).

CARVALHO-JÚNIOR AA, HENNEN JF (2008). Dicheirinia antunesii, a new rust species on the legume Ormosia from Brazil. Mycologia 100:776-778. (https://doi.org/10.3852/08-026).

CARVALHO-JÚNIOR AA, HENNEN JF (2009). Maravalia perae, a new species of rust fungus on Pera from Brazil. Mycologia 101:239-242. (https://doi. org/10.3852/08-154).

CARVALHO-JÚNIOR AA, HENNEN JF (2010). New species and nomenclature in Prospodium (Uropyxidaceae, Pucciniales). and the new anamorphic genus Canasta in the Neotropics. Mycologia 102:1096-1113. (https://doi.org/10.3852/09049).

CARVALHO-JÚNIOR AA, HENNEN JF (2012). The species of Puccinia on Piptocarpha and Vanillosmopsis in the Neotropics. Mycologia 104:557568. (https://doi.org/10.3852/11-141).

CARVALHO-JÚNIOR AA, HENNEN JF (2018). PUccinia on Ipomoea (Convolvulaceae) in Neotropics Phytotaxa 344:133-148. (https://doi. org/10.11646/phytotaxa.344.2.2).

CARVALHO-JÚNIOR AA, HENNEN JF (2019). Species of Puccinia on Malpighiaceae in Brazil. Phytotaxa 409:202-214.( https://doi.org/10.11646/ phytotaxa.409.4.2).

CARVALHO-JÚNIOR AA, HENNEN JF, HENNEN MM, FIGUEIREDO MB (2008). Fungos causadores de ferrugens (Uredinales) em áreas de Cerrado no Estado de São Paulo. Rodriguésia 59:1-55. (https://doi.org/10.1590/2175-7860200859101).

CAVALCANTI RB, JOLY CA (2002). Biodiversity and
Conservation Priorities in the Cerrado Region. In: Oliveira PS, Marquis, RJ (Eds.) The cerrados of Brazil: ecology and natural history of a neotropical savanna. New York Columbia University Press, pp. 351-368.

CHUPP C (1953). A monograph of the genus Cercospora. Cornell University, Ithaca, New York. 667 p. (https://doi.org/10.1093/aibsbulletin/4.3.11-d).

CONCHA QUEZADA, H. 2012. Anders Fredrik Regnell (1807-1884). Médico, botanista y mecenas en Suecia y Brasil. RIL editores, Santiago de Chile. 226p.

COOKE MC (1892). New exotic fungi. Grevillea 20:90-92.

COSTA AS (1986). Ahmés Pinto Viégas 1905-1986. Fitopatologia Brasileira 11:15-16.

CUMMINS GB, HIRATSUKA Y (1983). Illustrated genera of rust fungi. 2nd ed. The American Phytopathological Society, St. Paul. 152p.

CUMMINS GB, HIRATSUKA Y (2003). Illustrated genera of rust fungi. 3rd ed. The American Phytopathological Society, St. Paul. 225p.

DIANESE JC (2000). Micodiversidade associada a plantas nativas do Cerrado. In: Cavalcanti TB, Walter BMT. (Org.). Tópicos atuais em Botânica. 1ed. Brasília: Soc. Bras. Botânica / EMBRAPA, pp: 109-115.

DIANESE AC, VALE HMM, SOUZA ESC, PEREIRA-CARVALHO RC, CHAVES ZM, CÂMARA, PEAS, DIANESE JC (2014). New Cercospora species on Jatropha curcas in central Brazil. Mycological Progress 13:1069-1073. (ttps://doi.org/10.1007/ s11557-014-0994-y).

DIANESE AC, COSTA AM, DIANESE JC (2008). A new Pseudocercospora species on Passiflora setacea. Mycotaxon 105:1-5.

DIANESE JC (2014). Capítulo 11:A Implantação da Pós-Graduação em Ecologia. In: Diniz IR. (Org.). Instituto de Ciências Biológicas:1963-20013-Fragmentos de memórias. 1ed. Brasília: Editora Universidade de Brasília. pp:251-273.

DIANESE JC, BURITICÁ P, HENNEN JF (1994a). The rust of jatobá: a new Crossopsora species from Neotropica on Hymenaea (Leguminosae). Fitopatologia Brasileira 19:588-591.

DIANESE JC, CAFÉ FILHO AC (2014). Capítulo 6:História da Fitopatologia. In: Diniz IR. (Org.). Instituto de Ciências Biológicas:1963-20013-Fragmentos de memórias. 1ed. Brasília, DF: Editora Universidade de Brasília. pp:141-167. 
DIANESE JC, DIANESE AC (1995). Three Uncinula species from the Brazilian Cerrado and a key to South American Uncinula species. Mycological Research 99:821-824. (https://doi.org/10.1016/ S0953-7562(09)80733-8).

DIANESE JC, MEDEIROS RB, SANTOS LTP (1992). Cerradoa palmaea Hennen \& Ono found in two new hosts, Syagrus commosa (Mart.) Mart. and S. flexuosa Becc. Fitopatologia Brasileira 17:198. (https://doi.org/10.1080/00275514.1978.12020 260).

DIANESE JC, MEDEIROS RB, SANTOS LTP (1993a). Coniella costae sp. nov. on leaves of Myrcia tomentosa from the Brazilian cerrado. Mycological Research 97:1234-1236. (https://doi. org/10.1016/S0953-7562(09)81291-4).

DIANESE JC, MEDEIROS RB, SANTOS LTP (1997). Biodiversity of microfungi found on native plants of the Brazilian Cerrado. In: Hyde K. (Ed.). Biodiversity of tropical microfungi: Hong Kong University Press: Hong Kong, pp. 367-417.

DIANESE JC, MORAES TS, SILVA AR (1983). Response of thirteen species of Eucalyptus to field infection by Puccinia psidii Winter. In: IV Intern. Congress of Plant Pathology-1983, Melbourne, 200p.

DIANESE JC, MORAES TS, SILVA AR (1984). Response of Eucalyptus species to field infection by Puccinia psidii. Plant Disease 68:314-316. (https://doi.org/10.1094/PD-68-314)

DIANESE JC, SUTTON BC, TESSMAN DJ (1993b). Two deutromycetes, Phloeosporella flavio-moralis sp. nov. and Pseudocercospora punctata comb. nov. causing leaf lesions on Eugenia spp. Mycological Research 97:123-126. (https://doi. org/10.1016/S0953-7562(09)81169-6).

DIANESE JC, TESSMANN TJ, FURLANETTO C (1994c). Reinstating Oswaldina icarahyensis Rangel as the name of the anamorph of Apiosphaeria guaranitica. Sydowia, 46:33-237.

DIANESE JC, CÂMARA MPS (1994). Pseudocercospora aspidospermatis, a new combination for Bactrodesmiella aspidospermatis. Sydowia 46:225-232.

DIANESE JC, FURLANETTO C, SANTOS LTP (1999). Pseudocercospora zeyhera, a new combination for Cercospora zeyrae. Mycological Research 103:40-42. (https://doi.org/10.1017/ S0953756298007047).

DIANESE JC, INÁCIO CA, DORNELO-SILVA D (2001).
Wilmia, a new genus of phaeosphaeriaceous ascomycetes on Memora pedunculata in Central Brazil. Mycologia 93:1014-1018. (https://doi.org /10.1080/00275514.2001.12063234).

DIANESE JC, MEDEIROS RB, SANTOS LTP (1993c). Phloeosporella kitajimae sp. nov. associated with leaf spots and blight of Eugenia dysenterica. Mycological Research 97:610-612. (https://doi. org/10.1016/S0953-7562(09)81185-4).

DIANESE JC, PEREIRA-CARVALHO RC, INÁCIO CA (2010). Plurispermiopsis: a new capnodiaceous genus from the Brazilian Cerrado. Mycologia 102:1163-1166. (https://doi.org/10.3852/09253).

DIANESE JC, SANTOS LTP (1995). Aplopsora hennenii sp. nov. the first rust fungus recorded in host-family Vochysiaceae. Mycological Research 99:914-916. (https://doi.org/10.1016/S09537562(09)80749-1).

DIANESE JC, SANTOS LTP, MEDEIROS RB (1995). Kimuromyces cerradensis gen. et sp. nov., the rust of Gonçalo Alves. Fitopatologia Brasileira 20:251-255.

DIANESE JC, SANTOS LTP, MEDEIROS RB, SANCHEZ M (1993d). Batistopsora gen. nov. and new Phakopsora, Cerotelium, and Skierka species from the Brazilian cerrado. Fitopatologia Brasileira 18:436-450.

DIANESE JC, SANTOS LTP, MEDEIROS RB, SANCHEZ M (1994d). Mimema venturae sp. nov. on Dalbergia miscolobium in Brazlândia, Distrito Federal, Brazil. Mycological Research 98:786-788. (https://doi.org/10.1016/S0953-7562(09)810563).

DIANESE JC, SANTOS LTP (2010). Anais VI Congresso Brasileiro de Micologia. Brasília. Sociedade Brasileira de Micologia. 810p. Available at: http://livrozilla.com/doc/757083/anais-do-congresso---sociedade-brasileira-de-fitopatologia. Accessed on September 23, 2021.

DIETEL P (1897). Uredineae brasilienses a cl. E. Ule lectae. Hedwigia 36:26-37.

DIETEL P (1899). Uredineae brasilienses a cl. E. Ule lectae. II. Hedwigia 38:248-259.

DINIZ IR, DIANESE JC (2014). Capítulo 1:História do instituto de ciências biológicas e seus personagens. In: Diniz IR. (Org.). Instituto DE Ciências Biológicas:1963-20013-Fragmentos de memórias. 1ed. Brasília, DF: Editora Universidade DE Brasília. pp:15-76. 
DORNELO-SILVA D, DIANESE JC (2004). New hyphomycete genera on Qualea species from the Brazilian cerrado. Mycologia 96:879-884. (https://doi.org/10.1080/15572536.2005.1183293 4).

DORNELO-SILVA D, DIANESE JC (2003). Hyphomycetes on Vochysiaceae from the Brazilian Cerrado. Mycologia 95:1239-1251. (https://doi. org/10.1080/15572536.2004.11833032).

DORNELO-SILVA D, PEREIRA-CARVALHO RC, DIANESE JC (2007). New Stenella and Parastenella species from the Brazilian Cerrado. Mycologia 99:753-764. (https://doi.org/10.1080/15572536 .2007.11832538).

FARR ML (1973). An annoted list of Spegazzini's fungus taxa. Bibliotheca Mycologica. 35, Vols.1. and 2. Cramer, Lehre. $1661 \mathrm{pp}$.

FERNÁNDEZ-WINZER L, BERTHON KA, CARNEGIE AJ, PEGG GS, LEISHMAN MR (2019). Austropuccinia psidii on the move: survey-based insights to its geographical distribution, host species, impacts and management in Australia. Biol Invasions 21:1215-1225. (https://doi.org/10.1007/ s10530-018-1891-0).

FERREIRA FA (1989). Patologia Florestal. Principais doenças florestais no Brasil. Universidade Federal de Viçosa. 570 pp.

FIDALGO O (1968). Introdução à história da micologia Brasileira. Rickia 3:1-44.

FIGUEIREDO MB, HENNEN JF (1998). Uredinales (Ferrugens no Brasil e no Estado de São Paulo. Biológico 60:17-29.

FIRMINO AL, VÉLEZ-ZAMBRANO, SM, BARRETO RW, DIANESE JC (2019). Fungi from the "Lost World": Novel Asterinaceae from the Ricardo Franco Hills (Brazil), with a worldwide key to Lembosia species on Melastomataceae. Phytotaxa 409:129-145. (https://doi.org/10.11646/phytotaxa.409.3.2).

FIRMINO AL, INÁCIO CA, PEREIRA OL, DIANESE JC (2016). Additions to the genera Asterolibertia and Cirsosia (Asterinaceae, Asterinales), with particular reference to species from the Brazilian Cerrado. IMA Fungus 9:1-28. (https://doi. org/10.5598/imafungus.2016.07.01.02).

FUNDAÇÃO CASA DE RUI BARBOSA (2021). Galziou, o paisagista do Império. Available at: http:// www.casaruibarbosa.gov.br/glaziou/biografia. htm. Accessed on September 23, 2021.

FURLANETTO C, DIANESE JC (1997). Some Coe- lomycetes from Central Brazil. Mycological Research 102:19-29. (https://doi.org/10.1017/ S0953756297004656).

FURLANETTO C, DIANESE JC (1999). Some Pseudocercospora speceis and a new Prathigada species. Mycological Research 103:1203-1209. (https://doi.org/10.1017/S0953756299008394).

GALVÃO-ELIAS S, EBINGHAUS M, BEGEROW D, DIANESE JC (2918). Morphological review and phylogeny of Cerradoa palmaea, the rust fungus of Arecaceae from the Brazilian Cerrado. 11th International Mycol. Congress 2018, San Juan, Programe Book 1:281.

GARDNER G (1840). XXVI. Description of a new phosphorescent species of Agaricus. By George Gardner; with remarks upon it by the Rev. M. J. Berkeley. J. Bot. (Hooker) 2:426-428.

GARDNER G (1849). Travel in the interior of Brazil, During the years 1836-1841. $2^{\text {nd }}$ Ed. London: Reeve, Benham, and Reeve. 428p. Available at: https://books.google.com.br/ books?hl=pt- $\quad$ BR\&lr=\&id=nANMAAAAYAA$\mathrm{J} \& \mathrm{o} i=\mathrm{fnd} \& \mathrm{pg}=\mathrm{PA} 1 \& \mathrm{dq}=\mathrm{George}+$ Gardner, +who+lived+in+Brazil+from+1836+to+1841\&ot$\mathrm{s}=\mathrm{m} 2 \mathrm{D} 5 \mathrm{wu} 4 \mathrm{Mlj} \& \mathrm{sig}=6 \mathrm{QUR} 99 \mathrm{GCdRwmdDKy}$ V8J0- CVggy8\#v=onepage \&q=George\%20 Gardner\%2C\%20who\%20lived\%20in\%20Brazil\%20from\%201836\%20to\%201841\&f=false

GARDNER G (2020). Botanical exploration in Brazil. George Gardner's collections. Available at: http://www.kew.org/science/tropamerica/gardner/collections.html and http://www.kew.org/ science/tropamerica/gardner/bibliography.html. Accessed on March 9, 2020.

GIULIETTI AM, HARLEY RM, DE QUEIROZ LP, WANDERLEY MDGL, VAN DEN BERG C (2005). Biodiversity and conservation of plants in Brazil. Conservation Biology 19: 632-639. (https://doi. org/10.1111/j.1523-1739.2005.00704.x)

GIULIETTI AM, RAPINI A, ANDRADE MJG, DA SILVA JMC. (2009). Plantas raras do BrasilBelo Horizonte: Conservação Internacional 496 p. Avaiable at: https://www.conservation.org/docs/default-source/brasil/Conteudo-completo_Plantas-Raras-do-Brasil_final.pdf. Accessed on October 6, 2021.

GLOBAL PLANTS (2021). Ule, Ernst Heinrich Georg (1854-1915). Available at: https://plants.jstor. org/stable/10.5555/al.ap.person.bm000008665. Accessed on September 23, 2021. 
GLOBAL PLANTS (2021). Usteri, Alfred (18691948). Available at: https://plants.jstor.org/stable/10.5555/al.ap.person.bm000008686. Accessed on September 23, 2021.

GRILLO HVS (1935). A evolução da phytopathologia. Rodriguésia 1:1-12. Available at: http:// rodriguesia.jbrj.gov.br/FASCICULOS/Revistas\%20escaneadas\%20pela\%20Biblioteca/ per144398_1935_001_003.pdf. Accessed on September 27, 2021.

GUTERRES, DC, GALVÃO-ELIAS, S, DOS SANTOS, MDDM, DE SOUZA, BCP, DE ALMEIDA, CP, PINHO, DB, ... \& DIANESE, JC (2019). Phylogenetic relationships of Phaeochorella parinarii and recognition of a new family, Phaeochorellaceae (Diaporthales). Mycologia, 111: 660-675. (https://doi.org/10.1080/00275514.2019.1603025 ).

GUY N, BARRY M (2017). Response underway following myrtle rust find. Available at: https:// beehive.govt.nz/release/response-underway-following-myrtle-rust-find. 5 May 2017. Accessed on September 26, 2021.

GUTERRES DC, GALVÃO-ELIAS S, SOUZA, BCP, PINHO DB, SANTOS MDM, MILLER RNG, DIANESE JC (2018). Taxonomy, phylogeny, and divergence time estimation for Apiosphaeria guaranitica, a Neotropical parasite on bignoniaceous hosts. Mycologia 110:526-545. (https://doi.org/10.108 0/00275514.2018.1465774).

HENNEN JF, FIGUEIREDO MB, CARVALHO-JUNIOR AA, HENNEN PG (2005). Catalogue of plant rust fungi (Uredinales) of Brazil. Jardim Botânico do Rio de Janeiro, Rio de Janeiro, 490 pp. Available at: https://www.gov.br/jbrj/pt-br/centrais-de-conteudo/publicacoes/catalogue.pdf. Accessed on March 08, 2021.

HENNEN, JF, Y ONO (1978). Cerradoa palmaea: the first rust fungus on Palmae. Mycologia 70(3): 569-576.

HENNINGS PC (1895). Fungi Goyazenses. Hedwigia 34:88-116.

HENNINGS PC (1900). Fungi Mattogrossenses a DR.

R. Pilzer collecti 1899. Hedwigia 39:134-139.

HENNINGS PC (1902a). Fungi S. Paulenses I. a cl. Puttemans collecti. Hedwigia 41:104-118.

HENNINGS PC (1902b). Fungi S. Paulenses II. a cl. Puttemans collecti. Hedwigia 41:295-311.

HENNINGS PC (1904a). Fungi S. Paulenses III a cl. Puttemans collecti. Hedwigia 43:197-209.
HENNINGS PC (1904b). Fungi S. Paulenses IV a cl. Puttemans collecti. Hedwigia 48:1-20.

HENNINGS PC (1908). Fungi Bahienses a cl. Ule collecti. Hedwigia 47:266-270.

HENNINGS PC (1896). Beiträge zur Pilzflora Südamerikas I. Myxomycetes, Phycomycetes, Ustilagineae und Uredinae. Hedwigia 35:207-262.

HERNÁNDEZ-GUTIÉRREZ A, DIANESE JC (2009). New cercosporoid fungi from the Brazilian Cerrado 2. Species on hosts of the subfamilies Caesalpinioideae, Faboideae, and Mimosoideae (Leguminosae s. lat.). Mycotaxon 107:1-24. (https:// doi.org/10.5248/107.1).

HERNÁNDEZ-GUTIÉRREZ A, DIANESE JC (2014a). New Passalora species on Peixotoa (Malpighiaceae) from the Brazilian Cerrado. Mycological Progress 13:75-79. (https://doi.org/10.1007/ s11557-013-0894-6).

HERNÁNDEZ-GUTIÉRREZ A, BRAUN U, DIANESE, JC (2014). Cercosporoid hyphomycetes on malpighiaceous hosts from the Brazilian Cerrado: species of Pseudocercospora on hosts belonging to Byrsonima. Mycological Progress 13:193-210. (https://doi.org/10.1007/s11557-013-0922-6).

HERNANDEZ-GUTIERREZ A, CHAVES ZM, DORNELO-SILVA D, DIANESE JC (2015). Additions to the cercosporoid fungi from the Brazilian Cerrado: New species on hosts belonging in family Fabaceae, and reallocations of four Stenella species into Zasmidium. Mycobiota 5:33-64. (https://doi. org/10.12664/mycobiota.2015.05.06).

HERNÁNDEZ-GUTIÉRREZ A, DIANESE JC (2008). New cercosporoid fungi from the Brazilian Cerrado 1. Species on hosts of the families Anacardiaceae. Mycotaxon 106:41-63.

HERNÁNDEZ-GUTIÉRREZ A, DIANESE JC. (2014b). Cercosporoid hyphomycetes on malpighiaceous hosts from the Brazilian Cerrado:New Passalora and Pseudocercospora species on hosts of the genus Banisteriopsis. Mycological Progress 13:365371. (https://doi.org/10.1007/s11557-013-0922$6)$.

INÁCIO CA, PEREIRA-CARVALHO RC, SOUZA ESC, SALES HB, DIANESE, JC (2012). A new Hysterostomella species from the Cerrado in Brasília National Park. Mycotaxon 119:307-313. (https:// doi.org/10.5248/119.307).

INÁCIO CA, DIANESE JC (1998). Foliicolous fungi on Tabebuia species. Mycological Research 102, n.102:695-708. (tps://doi.org/10.1017/ 
S0953756297005856).

INÁCIO CA, DIANESE JC (1999). A new Mycovellosiella species on Myracrodruon urundeuva. Mycotaxon, Ithaca, New York 72:251-254.

INÁCIO CA, DIANESE JC (2006). Foliicolous fungi on Tabebuia species from the cerrado. Mycological Progress 5:120-127. (https://doi.org/10.1007/ s11557-006-0507-8).

INÁCIO CA, FURLANETTO C, GUTIERRES AH, DIANESE JC (1996). Some Cercospora species originally described by Ahmés Pinto Viégas. Fitopatologia Brasileira 21:405-409.

INÁCIO CA, PEREIRA-CARVALHO RC, SOUZA, ESC, DIANESE JC (2011) A new Dothidasteroma species on leaves of Psidium laruotteanum from the Brazilian Cerrado. Mycotaxon 116:27-32. (https://doi.org/10.5248/116.27).

JACKSON HS (1926). The Rusts of South America Based on the Holway Collections. I. Mycologia 18:139-162. (https://doi.org/10.1080/00275514 .1926.12020503).

JACKSON HS (1927). The rusts of South America based on the Holway collections 11. Mycologia 19:51-65. (https://doi.org/10.1080/00275514.1 927.12020529).

JACKSON HS (1931a). The Rusts of South America Based on the Holway Collections III. Mycologia 23:96-116. (https://doi.org/10.1080/00275514.1 931.12017027).

JACKSON HS (1931b). The Rusts of South America Based on the Holway Collections IV. Mycologia 23:332-364. (https://doi.org/10.1080/00275514 .1931.12017049).

JACKSON HS (1931c). The rusts of South America based on the Holway collections V. Mycologia 23:463-503. (https://doi.org/10.1080/00275514 .1931.12017056)

JACKSON HS (1932). The rusts of South America based on the Holway collections VI. Mycologia 24:62-186. (https://doi.org/10.1080/00275514.1 932.12020608).

JARDIM BOTÂNICO DE BRASÍLIA (2021). Herbário Ezechias Paulo Heringer. Available at: https:// www.jardimbotanico.df.gov.br/pesquisa/herbario-eph/. Accessed on September 26, 2021.

JØRSTAD I (1956). Uredinales from South America and tropical North America, chiefly collected by Swedish botanists. Arkiv für Botanik (serie 2) 3:443-490.

JUEL HO (1897). Die Ustilagineen und Uredineen der erstn Ragnellschen Expedition. Beih. Svenska Vet.-Akad. Handl. 23:1-30.

LIMA JEFW. (2011). Situação e perspectivas sobre as águas do cerrado. Ciência e Cultura 63: 27-29. (http://dx.doi.org/10.21800/S000967252011000300011).

LINK HF (1809). Observationes in ordines plantarum naturales. Ges. Naturf. Freunde Berlin Mag. 3:342.

MARTINELLI G, \& MORAES MA. (2013). Livro vermelho da flora do Brasil. $1^{\text {a }}$ Edição. Rio de Janeiro : Andrea Jakobsson: Instituto de Pesquisas Jardim Botânico do Rio de Janeiro. 104 p. Available at: https://www.jakobssonestudio.com.br/livro/livro-vermelho-da-flora-do-brasil. Accessed on October 06, 2021.

MARTINS-JUNIOR AS, SAKURAGUI CM, HENNEN JF, \& JUNIOR AAC (2019). Neopuccinia (Pucciniales): A new Puccinia-like genus from the Brazilian Cerrado. Phytotaxa, 406(3), 169-179. (https:// doi.org/10.11646/phytotaxa.406.3.3).

MARTINELLI G, MESSINA T, SANTOS-FILHO L (ORG.) (2014) Livro Vermelho da Flora do Brasil, Plantas Raras do Cerrado. Rio de Janeiro : Instituto Jardim Botâncio, CNCFlora. 320 p.

MAUBLANC A (1913). Rapport sur les maladies observées au Laboratoire de Phytopathologie du Musée National de Rio de Janeiro. Bulletin Mensuel des Renseignements Agricoles et des Maladies des plantes. Rome 4:876-879.

MAUBLANC A, RANGEL E (1915). Alguns fungos do Brasil, novos ou mal conhecidos. Boletim de Agricultura 16:310-328.

MEDEIROS RB, DIANESE JC (1994). Passalora eitenii sp. nov. on Syagrus comosa (Mart.) Mart. in Central Brazil and a key for identification of Passalora species. Mycotaxon, Ithaca, N. York, 51:509-513.

MENDONÇA RC, FELFILI JM, WALTER BMT, SILVA JUNIOR MC, FILGUEIRAS TS, NOGUEIRA PE, FAGG CW (2008). Flora vascular do bioma Cerrado: checklist com 12.356 espécies. In:S.M. Sano; Almeida, S.P. and J.F. Ribeiro (eds.). Cerrado: ecologia e flora, vol. 2. Embrapa Informação e Tecnologia, Brasília. p. 423-1279.

MITTERMEIER RA, MYERS N, THOMSEN JB, et al (1998). Biodiversity hotspots and major tropical wilderness areas: Approaches to setting conservation priorities. Conservation Biology 12:516-520. (https://doi.org/10.1046/j. 1523-1739.1998.012003516.x). 
MYERS N (2003). Biodiversity hotspots revisited. Bioscience 53:916-917. (https://doi.org/10.1641/0006-3568(2003)053[0916:BHR]2.0. CO;2).

MYERS N, MITTERMEIER RA, MITTERMEIER CG, et al. (2000). Biodiversity hotspots for conservation priorities. Nature 403:853-858. (https://doi. org/10.1038/35002501).

NAVARATNAM S (1985). Guava rust: Puccinia psidii Winter. Plant Quarantine Leaflet No. 45. Australia: Commonwealth Department of Primary Industry. $4 p$.

NYBG STEERE HERBARIUM (2021). Universidade Federal Rural do Rio de Janeiro. Available at: http://sweetgum.nybg.org/science/ih/herbarium-details/?irn=126374. Accessed on September 26, 2021.

PARQUE ECOLÓGICO EZECHIAS HERINGER (2021). Parque do Guará. Available at: https://www.guara.df.gov.br/category/sobre-a-ra/parques-do-guara/parque-ecologico-ezechias-heringer/. Accessed on: September 26, 2021.

PATRIMÔNIO BELGA NO BRASIL (2021). Puttemans, Arsène (1873-1937). Available at: http:// www.belgianclub.com.br/pt-br/creator/puttemans-ars\%C3\%A8ne-1873-1937. Accessed on September 23, 2021.

PEGG G, CARNEGIE A, GIBLIN F, PERRY S (2018). Managing myrtle rust in Australia. Canberra, Australia: Plant Biosecurity Cooperative Research Centre. 137p. Available at: http://era.daf.qld. gov.au/id/eprint/6163/1/2063\%20Final\%20Report\%20CRC\%202063\%20Pegg\%20etal\%202017. pdf. Accessed on September 26, 2021.

PEREIRA-CARVALHO RC, SEPULVEDA-CHAVERA G, ARMANDO EAS, DIANESE JC (2009a). An overlooked source of fungal diversity: novel hyphomycete genera on trichomes of Cerrado plants. Mycological Research 113:261-274. (https://doi.org/10.1016/j.mycres.2008.11.005).

PEREIRA-CARVALHO RC, DORNELO-SILVA D, INÁCIO CA, DIANESE JC (2009b). Chaetothyriomyes:a new genus in family Chaetothyriaceae. Mycotaxon 107:483-488. ( https://doi. org/10.5248/107.483).

PEGLER DN (1996). Advance in tropical mycology initiated by British mycologists. In: Sutton B. (ed) A century of mycology. Cambridge University Press, New York, pp 53-79.

RANGEL E (1916a). Contribuição para o estudo dos Puccinias das Myrtacéas. Archiv. Mus. Nac. 18:147-156.

RANGEL E (1916b). Fungos do Brazil, novos ou mal conhecidos. Archiv. Mus. Nac 18:157-164.

RANGEL E (1918). Alguns fungos novos do Brasil. Archivos do Jardim Botânico Rio de Janeiro 2:69-74.

RANGEL E (1921). Nota sobre dois fungos. Archiv. Escola Superior Agric. Medic. Veter. Niteroi 5:3539.

REZENDE DV, DIANESE JC (2003a). Espécies de Uromyces em leguminosas do Cerrado com descrição de $U$. galactiae sp. nov. Fitopatologia Brasileira 28:495-501. (https://doi.org/10.1590/ S0100-41582003000500005).

REZENDE DV, DIANESE JC (2001). New Ravenelia species on leguminous hosts from the Brazilian Cerrado. Fitopatologia Brasileira 26:627-634. (https://doi.org/10.1590/S010041582001000300008).

REZENDE DV, DIANESE JC (2002). Aspectos taxonômicos de Uredinales infectando leguminosas utilizadas na arborização urbana do Distrito Federal. Fitopatologia Brasileira 27:361-371. (https://doi. org/10.1590/S0100-41582002000400005).

REZENDE DV, DIANESE JC (2003b). Revisão taxonômica de algumas espécies de Ravenelia em leguminosas do Cerrado brasileiro. Fitopatologia Brasileira 28:27-36. (https://doi.org/10.1590/ S0100-41582003000100004).

SANTOS K (2016). Brazilian plant specimens at the Regnellian herbarium: history and repatriation. Rodriguésia 67:879-892. (https://doi. org/10.1590/2175-7860201667403).

SANTOS MDM, BOITEUX MEF, BOITEUX LS, SARAIVA PEA, DIANESE JC (2016). ITS-Phylogeny and taxonomy of Phyllachora species on native Myrtaceae from the Brazilian Cerrado. Mycologia 108:16-25. (https://doi.org/10.3852/16-025).

SANTOS MDM, SOARES WRO, ESC SOUZA, SILVA RAF GUTERRES DC, PINHO DB, DIANESE JC (2020). Cladosterigma: an enigmatic fungus, previously considered a basidiomycete, now revealed as an ascomycete member of the Gomphillaceae. Mycologia 112: 829-846. (https://doi.org/1 0.1080/00275514.2020.1781501).

SCARIOT A, SOUSA-SILVA JC, FELFILI JM (2005). Cerrado: ecologia, biodiversidade e conservação. Volume 1. Brasília: Ministério do Meio Ambiente.439p. Available at: http://biblioteca.cl.df.gov. br/dspace/bitstream/123456789/630/1/Cerra- 
do_Parte1.pdf. Accessed on October 10, 2021.

SILVA M, MINTER DW (1995). Fungi from Brazil, recorded by Batista and co-workers. Mycological Papers 169:1-585.

SOARES WRO, DIANESE JC. (2014). New Meliola species on fabaceous hosts from the Brazilian Cerrado. Mycological Progress 13:321-331. (https://doi.org/10.1007/s11557-013-0917-3).

SOCIEDADE BOTÂNICA DO BRASIL (2021). Diretorias Nacionais Anteriores. Available at: https://www.botanica.org.br/diretorias-nacionais-anteriores/ and https://docplayer.com. br/39643793-Ezechias-paulo-heringer-historia. html. Accessed on September 26, 2021.

SOUZA CAP, VITORIA NS, BEZERRA JL, LUZ EDMN, DIANESE JC, INÁCIO, CA (2008). Camarotella brasiliensis sp. nov. (Phyllachoraceae) on Syagrus schizophylla (Arecaceae) from Brazil. Mycotaxon 103:313-317.

SOUZA ESC (2016). Morfotaxonomia e filogenia de Pucciniales do Cerrado brasileiro. DSc Dissertation. Brasília: Universidade de Brasília. 350 p. Available at: https://repositorio.unb.br/handle/10482/31967. Accessed on September 2021.

SOUZA ESC, CHAVES ZM. SOARES WRO, PINHO DB, DIANESE JC (2015). Uromyces hawksworthii nom. nov. for Aecidium goyazense, on Phthirusa stelis (Loranthaceae) from the Brazilian Cerrado. IMA Fungus 6:155-162. (https://doi. org/10.5598/imafungus.2015.06.01.10).

SOUZA ESC, VALE HMM, PEREIRA CRC, SOARES WRO; MILLER RNG, DIANESE, JC (2017). Infection by Uromyces euphorbiae:a trigger for the sporulation of endophytic Colletotrichum truncatum on the common host Euphorbia hirta. Mycological Progress 16:941-946. (https://doi. org/10.1007/s11557-017-1338-5).

SOUZA ESC, AIME MC, GALVÃO-ELIAS S, PINHO DB, MILLER RNG, DIANESE JC (2018). Crossopsorella, a new tropical genus of rust fungi. Phytotaxa 375: 189-202. (https://doi.org/10.11646/ phytotaxa.375.3.1)

SPEGAZZINI CL (1881a). Fungi Argentini additis nonnullis Brasiliensibus Montevideensibusque. Pugillus Quartus. Anales de la Sociedad Científica Argentina 12:13-30.

SPEGAZZINI CL (1881b). Fungi Argentini additis nonnullis Brasiliensibus Montevideensibusque. Pugillus Quartus [cont.]. Anales de la Sociedad Científica Argentina 12:174-189.
SPEGAZZINI CL (1881c). Fungi Argentini additis nonnullis Brasiliensibus Montevideensibusque. Pugillus Quartus [cont.]. Anales de la Sociedad Científica Argentina 12:208-227.

SPEGAZZINI CL (1881d). Fungi Argentini additis nonnullis Brasiliensibus Montevideensibusque. Pugillus Quartus [cont.]. Anales de la Sociedad Científica Argentina 12:97-117.

SPEGAZZINI CL (1889). Fungi Puiggariani. Pugillus 1. Boletín de la Academia Nacional de Ciencias Córdoba 11:381-622.

SPEGAZZINI CL (1908). Fungi aliquot Paulistani. Rev. Mus. La Plata 15:7-48.

STRASSBURG BBN, BROOKS T, FELTRAN-BARBIERI R, IRIBARREM A, CROUZEILLES R, LOYOLA R, LATAWIEC AE, OLIVEIRA FILHO FJB, SCARAMUZZA CAM, SCARANO FR, SOARES-FILHO B, BALMFORD A (2017). Moment of truth for the Cerrado hotspot. Nature Ecology \& Evolution 1:13. (https://doi.org/10.1038/s41559-017-0099)

SUTHERLAND R, SOEWARTO J, BERESFORD R, GANLEY B (2020). Monitoring Austropuccinia psidii (myrtle rust) on New Zealand Myrtaceae in native forest. New Zealand Journal of Ecology 44:1-5. (https://dx.doi.org/10.20417/nzjecol.44.23).

TESSMANN DJ, DIANESE JC, MIRANDA AC, CASTRO LHR (2001). Epidemiology of a neotropical rust (Puccinia psidii): periodical analysis of the temporal progress in a perennial host (Syzygium jambos). Plant Pathology 50:725-731. (https:// doi.org/10.1046/j.1365-3059.2001.00646.x).

TEULON DA, ALIPIA TT, ROPATA HT, GREEN JM, VILJANEN-ROLLINSON SL, CROMEY MG, ARTHUR K, MACDIARMID RM, WAIPARA NW, MARSH AT (2015). The threat of myrtle rust to taonga plant species in New Zealand. New Zealand Plant Protection 68: 66-75. (https://doi. org/10.30843/nzpp.2015.68.5869).

TOOME-HELLER M, HO WWH, GANLEY RJ, ELLIOTT CEA, QUINN B, PEARSON HG, ALEXANDER BJR. 2020. Chasing myrtle rust in New Zealand: host range and distribution over the first year after invasion. Australasian Plant Pathology (https://doi. org/10.1007/s13313-020-00694-9).

THURSTON-JUNIOR HW (1940). The rusts of Minas Gerais, Brazil, based on collections by A.S. Muller. Mycologia 32:290-309. (https://doi.org/10.1080/ 00275514.1940.12017412).

UCHIDA J, ZHONG S, KILLGORE E (2006). First re- 
port of a rust disease on Ohia caused by Puccinia psidii in Hawaii. Plant Disease, 90: 524-524. (https://doi.org/10.1094/PD-90-0524C).

VELAZCO SJE (2018). O Cerrado Além Das Fronteiras: Padrões De Diversidade E Ameaças Presentes E Futuras À Sua Flora Na Bolívia, Brasil E Paraguai. DSc Dissertation. Curitiba: Universidade Federal do Paraná. 175 p. Available at: https://www.acervodigital.ufpr.br/bitstream/ handle/1884/58205/R\%20-\%20T\%20\%20SANTIAGO\%20JOSE\%20ELIAS\%20VELAZCO.pdf?sequence $=1$ \&isAllowed=y. Accessed on 06 October, 2021.

VIÉGAS AP (1943). Alguns fungos do Cerrado. Bragantia 3:49-62.

VIÉGAS AP (1944). Alguns fungos do Brasil. II. Ascomicetos. Bragantia 4:5-392.

VIÉGAS AP (1945a). Alguns fungos do Brasil. XI. Fungi Imperfecti (Sphaeropsidales). Bragantia 5:717779.

VIÉGAS AP (1945b). Alguns fungos do Brasil. IV. Uredinales. Bragantia 5:1-144.

VIÉGAS AP (1946a). Alguns fungos do Brasil. XII. Fungi Imperfecti (Melanconiales). Bragantia 6:1-
37.

VIÉGAS AP (1946b). Alguns fungos do Brasil. XIII. Hifomicetos. Bragantia 6:353-442.

VIÉGAS AP (1946c). Quatro ascomicetos novos Brasileiros. Boletim da Sociedade Brasileiro de Agronomia 9:1-4.

VIÉGAS AP (1945c). Alguns fungos do Brasil. Cercosporae. Boletim Soc. Bras. Agron. 8:1-160.

VIÉGAS AP (1947). Alguns fungos encontrados em S. Paulo, Minas e Espírito Santo. Bragantia 7:107124.

VIÉGAS AP (1960). Porotenus n. gen. Uredinearum. Bragantia 19:95-99.

VIÉGAS AP (1979). Dicionário de fitopatologia e micologia. Instituto Agronômico de Campinas. São Paulo. 890 p.

VIÉGAS AP (1961). Índice de fungos da América do Sul. Campinas: Seção de Fitopatologia, Instituto Agronômico, Campinas, p 912.

VIÉGAS AP, CARDOSO L (1944). Queirozia novo género da familia Erysiphaceae. Boletim da Sociedade Brasileira de Agronomia 7:1-6.

VIÉGAS AP, TEIXEIRA CG (1945). Alguns fungos de Minas Gerais. Rodriguésia 9:49-56. 